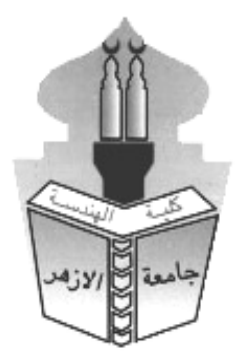

\title{
STRATEGIES OF BIO MIMICRY AS A TOOL FOR SUSTAINABILITY (APPLICATIONS IN SIMULATION FOR DESIGN COLLEGES' STUDENTS)
}

\author{
Mahmoud Tarek Mohamed Ahmed Hammad. \\ Architectural Engineering Dept., Faculty of Engineering, Al-Azhar University, Cairo, Egypt. \\ E-mail:Mahmoud.Hamad65@azhar.edu.eg
}

\begin{abstract}
Strategies of bio mimicry are considered one of the most modern and vital trends, which affecting the architectural thinking and have an effective role in increasing the building efficiency towards its surrounding environment. Nature has been and remained the source of systems and processes. Due to its rules and principles, it could preserve the ecosystem through which design solutions could be drawn. Such solutions are more capable than humans, and the design thinking which combines biology and architecture and creating integration between building and nature is called science of bio mimicry. Such phenomenon has assisted the architectural applications on generating sustainable shapes that are more balanced to the surrounding environment, however some of these applications are superficial and depended on simulation of living shapes with ignoring the biological relationship between such shapes with their environment. Consequently, the research dilemma is represented in not utilizing, implicitly and in depth, from natural science in preparing architectural designs whether such science is physical or biology of the ecosystems for living organism and their principles that ensuring its internal cohesion, which in turn adapting with the environmental changes surrounding. Therefore, the study highlights the significance of studying the strategies and principles of bio mimicry, and utilized them and thus it is possible to create innovative design solutions to achieve more sustainable ecological balance. By studying the simulation techniques and highlight at certain applications utilized by at design works, the research has emphasized the importance of the integration of biology with nature, and this attitude of understanding such ecological systems could lead to developing a methodology for a sustainable ecological design, of which the environmental biology shall become a vital element integrated with ecological design systems.
\end{abstract}

KEYWORDS: Bio mimicry, Building efficiency, the Ecological System, Biology, Integration, Architectural Applications, Nature Science, Environmental Balance, Sustainability.

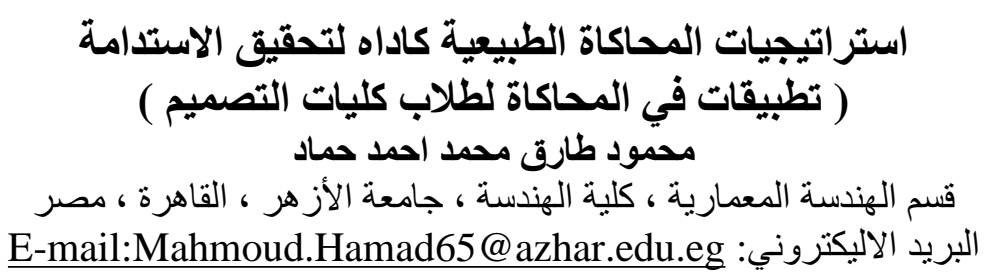

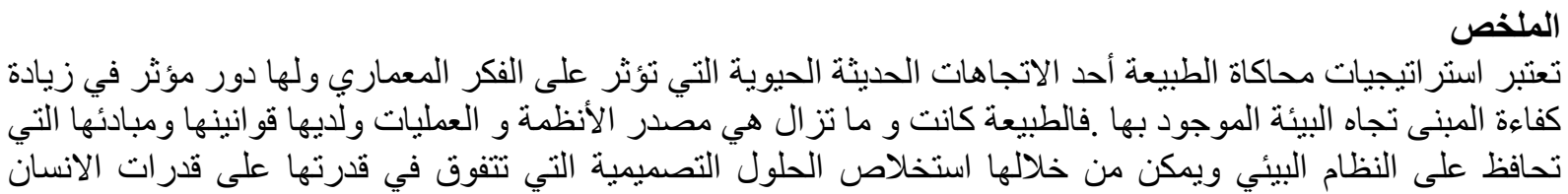


وويسمى الفكر التصميمي الذي يجمع بين علم الأحياءو العمارة ويحقق التكامل بين المبني و الطبيعة بعلم محاكاة الطبيعة .

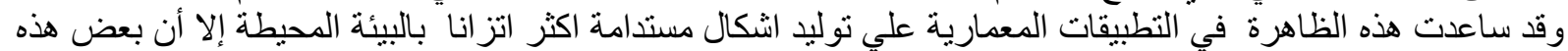

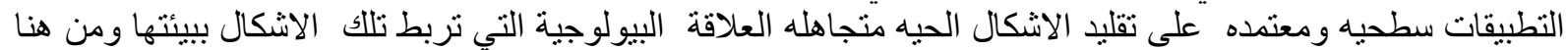

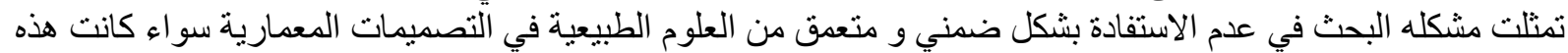

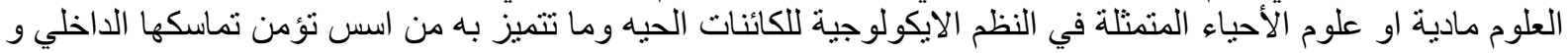

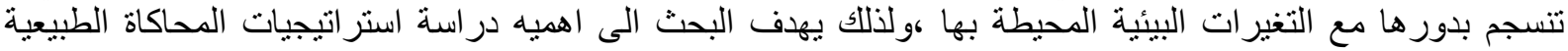

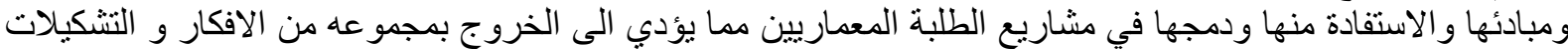

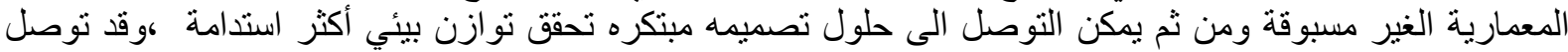

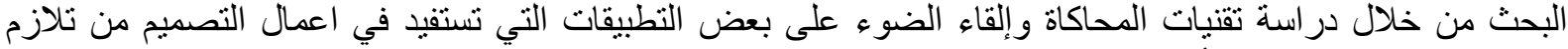

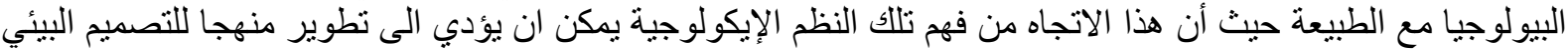

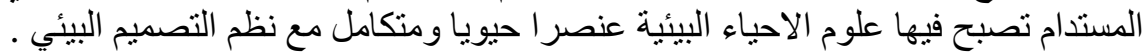

الكلمات المفتاحيه : محاكاة الطبيعة ، كفاعة المبني ، النظام البيئي ، علم الأحياء ، التكامل ، التطبيقات المعمارية ، العلوم الطبيعية ، توازن بيئي ، الاستدامة.

1 - (مقدمبه

ظهرت عماره المحاكاة الطبيعية كأحد الاتجاهات الحديثة للفكر المعماري الذي يهتم بالعلاقة بين المبنى وبيئته سواء كانت

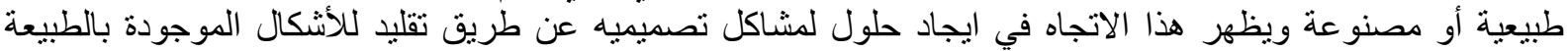

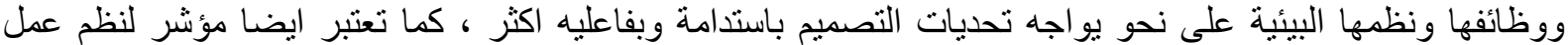

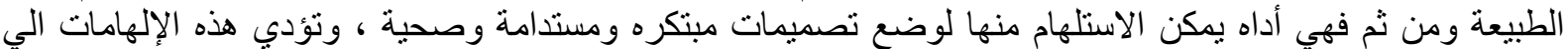

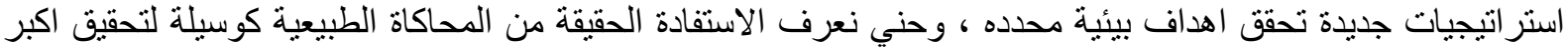

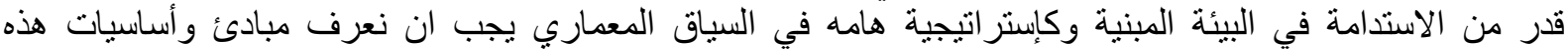

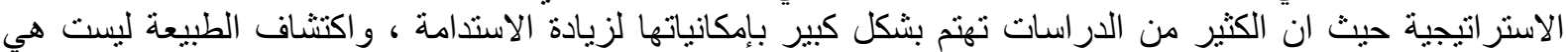

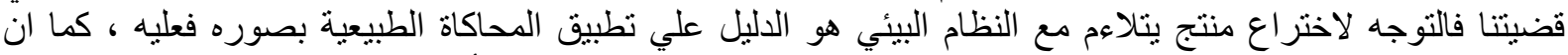

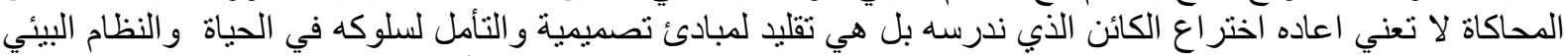

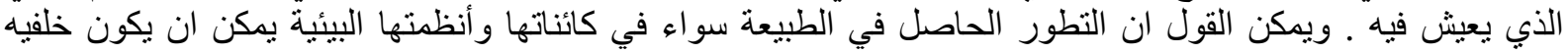
ومصدر و اعد في مجال التصميمات المعمارية وذلك بفضل المحاكاة التي تقوم بها بر امج الكمبيوتر .

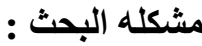
تظهر الثكاليه البحث في امكانه البكانيه الاستفادة من مبادئ وأفكار عماره المحاكاة الطبيعية كأحد الاتجاهات الحديثة وكنهج

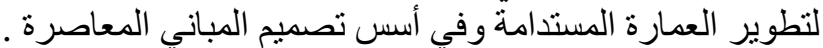

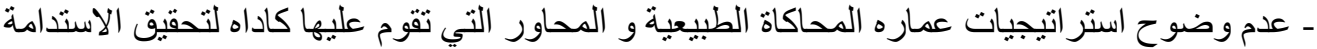

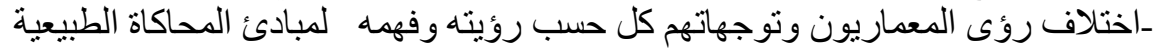

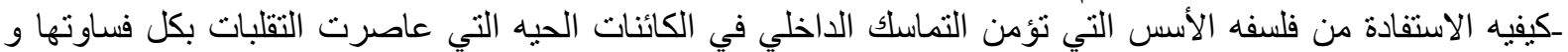

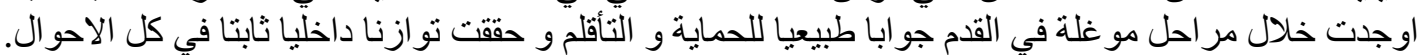

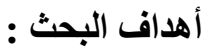

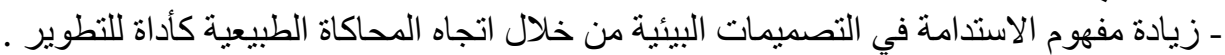

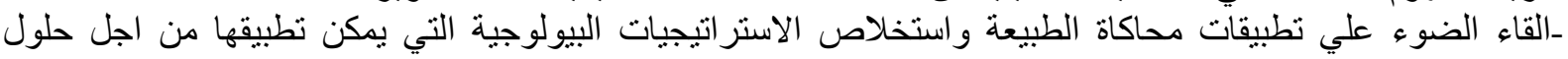
تصميمية مبتكرة في مجالات التصميم و العمارة .

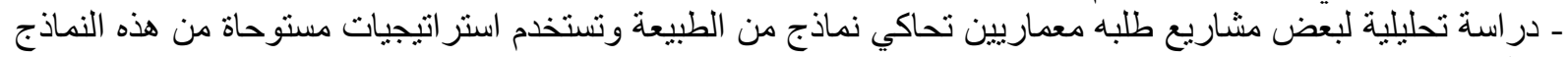

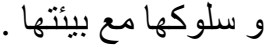

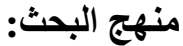

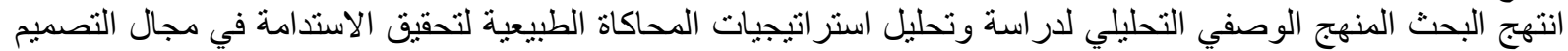

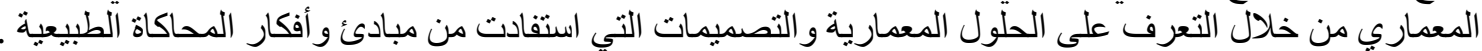

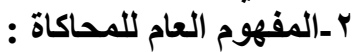

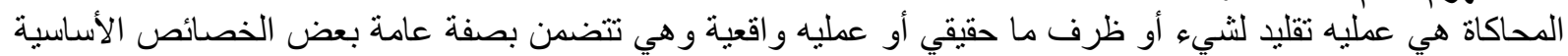

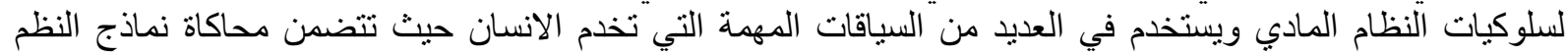

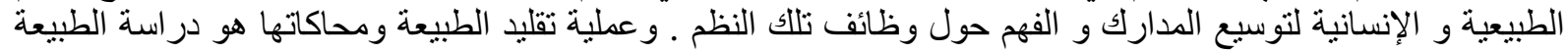
في النماذج و النظم و العمليات حتى تستلهم منها ما يحل مشاكل الانسان ويمكن ان يكون تقليد مستوى مستلهم بيولو جيا وهو

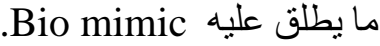
ويمكن القول ان علم محاكاة الطبيعة يجمع بين العديد من العلوم التطبيقية كالفيزياء و الكيمياء و العلوم الهندسية و علم

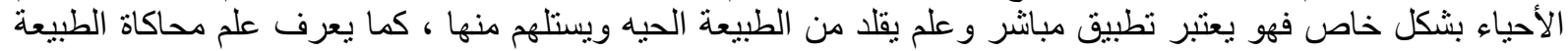


بأنه اختيار للطبيعة ولنماذجها و أنظمتها و عملياتها و عناصر ها لكي تناقش من قبل البشر و تعالج بالأحياء من اجل مشاكل

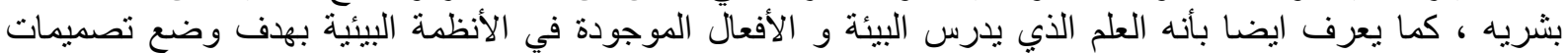
منو ازنة مع البيئة.

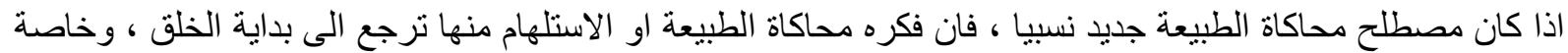

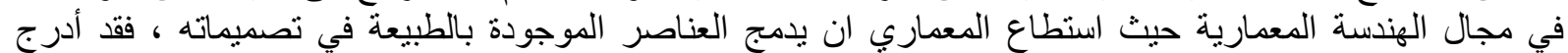

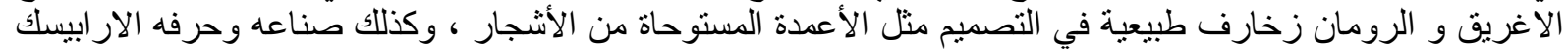

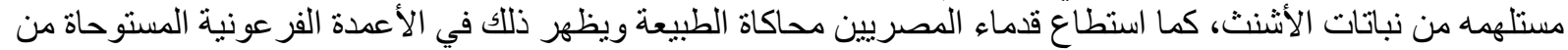

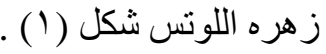
لقد تعلم الانسان في جميع الثقافات المختلفة كيفيه البقاء على الحياة من الكائنات الحيه المحيطة بهاه و اعتبر ها نماذج مثاليه من

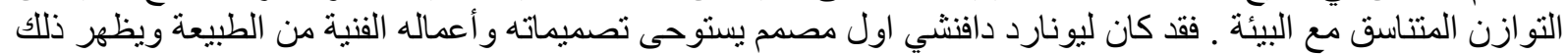

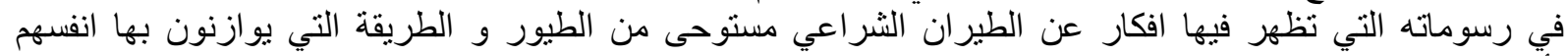

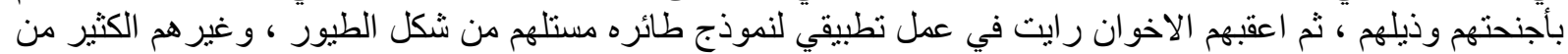

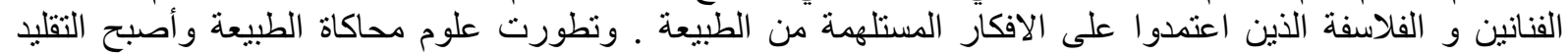

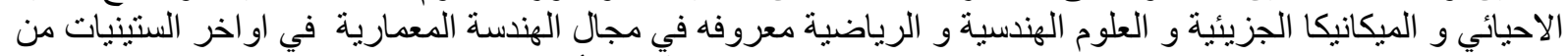

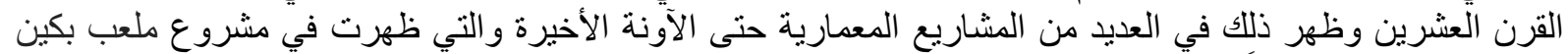

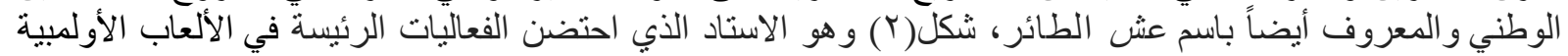
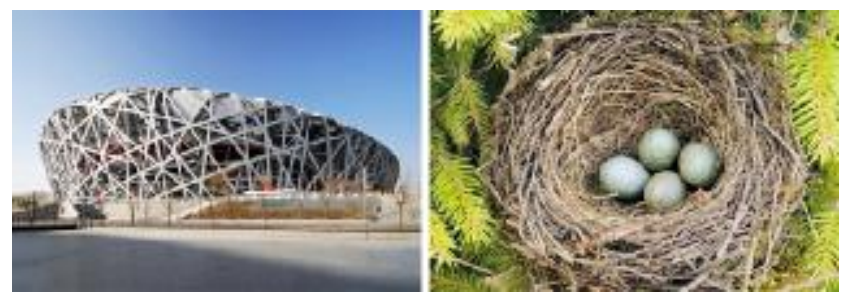

شكل (r) يوضح ملعب بكين الوطني ويعرف أيضاً باسم عش الطائر

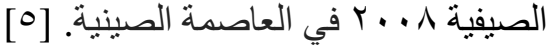

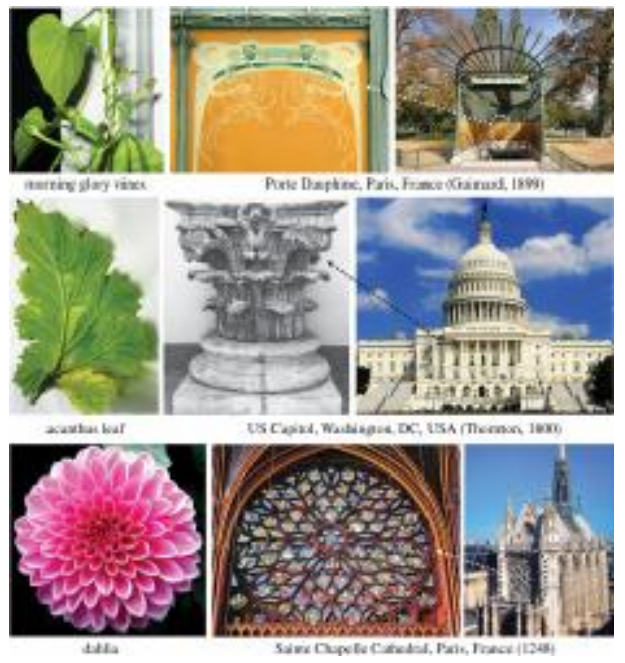

شكل ( ) ) يوضح نماذج بناء قديما مستوحاة من

الكائنات الحيه المحيطة

ع ـاستراتيجيات محاكاة الطبيعة :

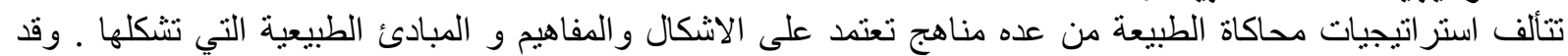

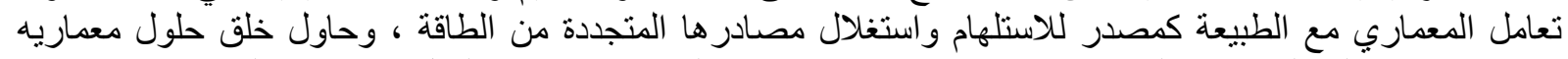

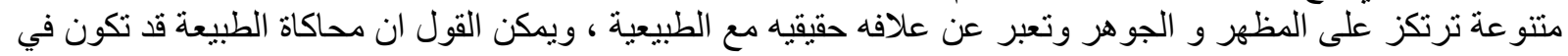

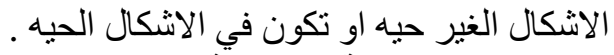

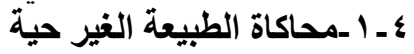

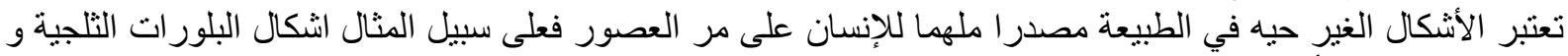

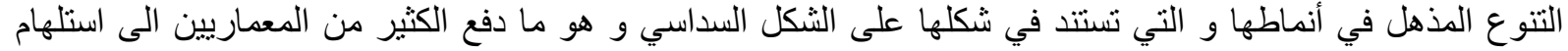

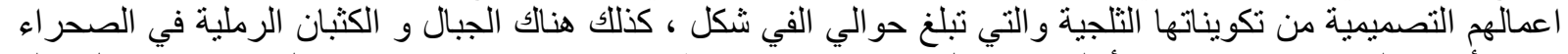

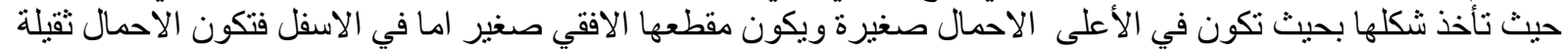

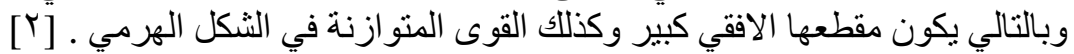
ع ـ ـ ـمحاكاة الطبيعة الحية

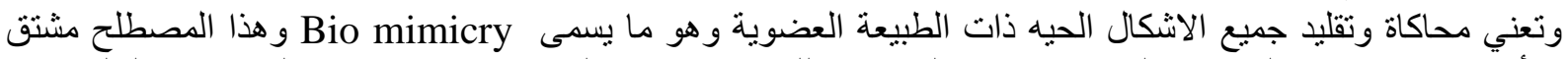

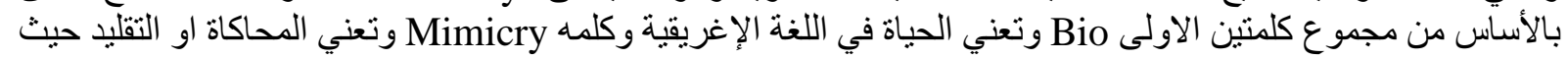




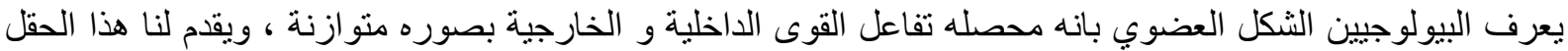

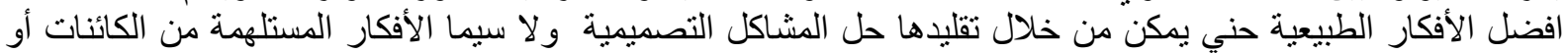

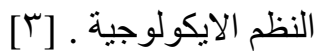

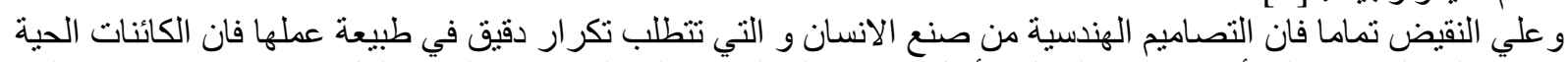

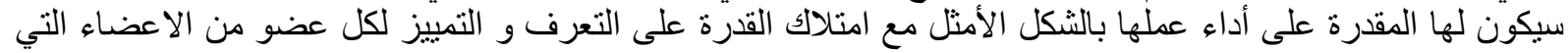

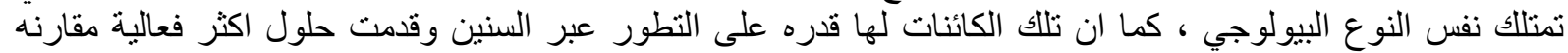

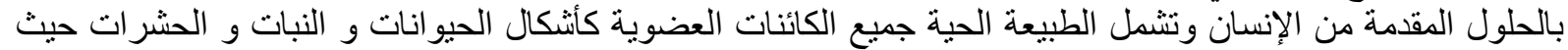

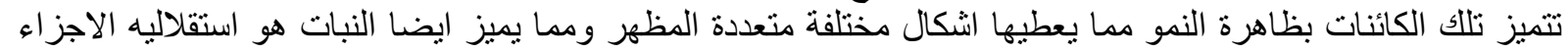

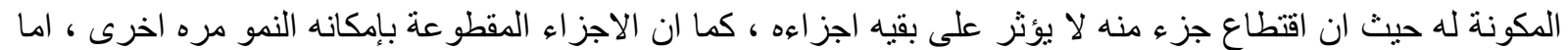

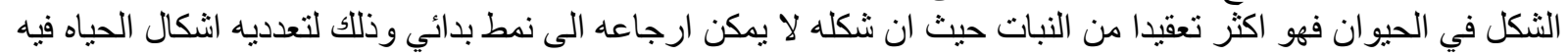

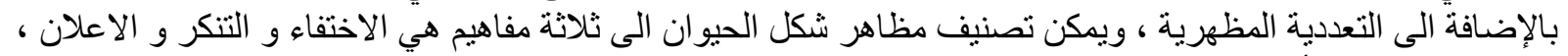

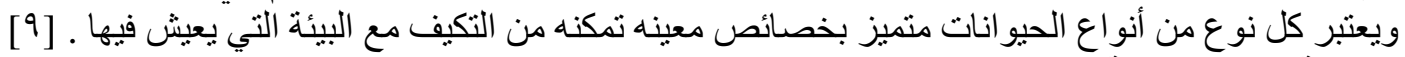

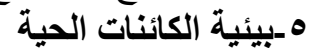

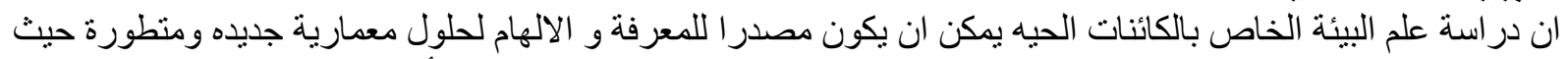

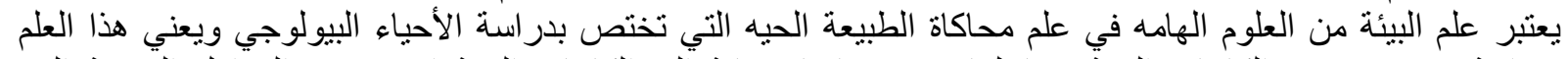

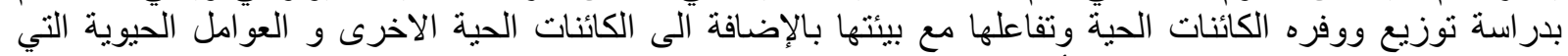

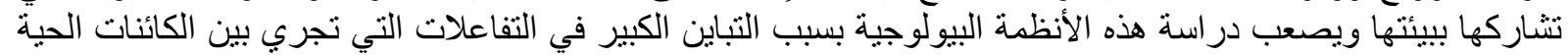
المختلفة و حتى الكائنات الدقيقة كالبكتيريا يمكن ان تستجيب لبيئتها بقدر ما يستجيب الاسد الباحث عن طعامه في السافانا .

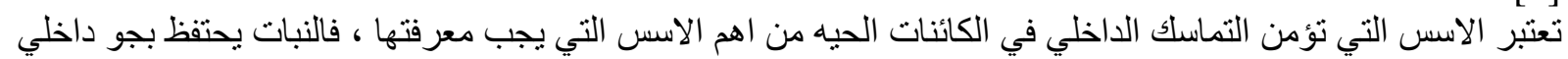

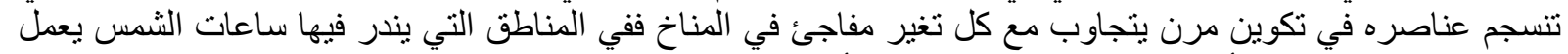

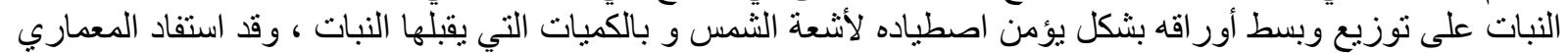

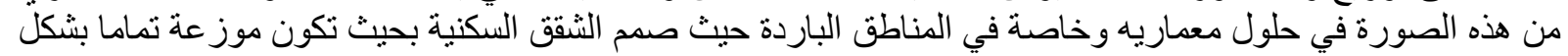

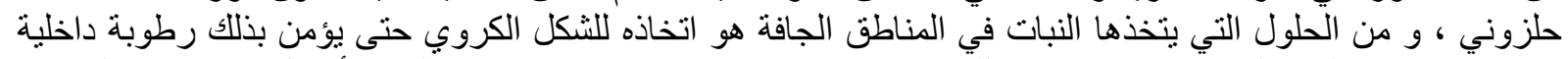

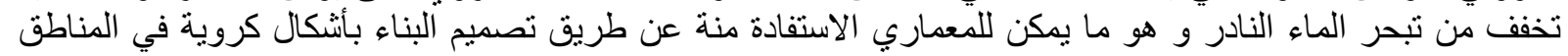

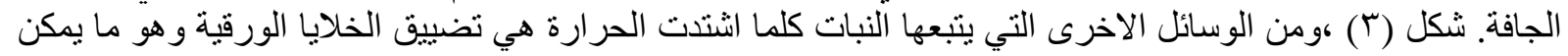

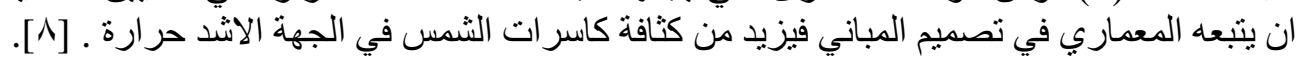

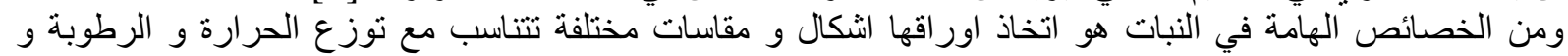

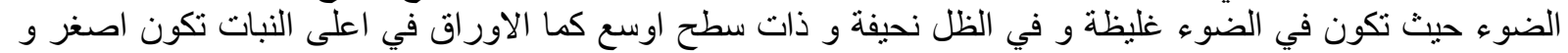

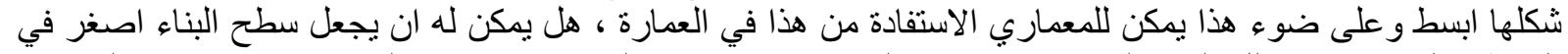

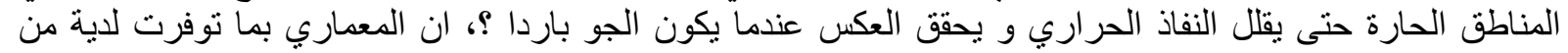

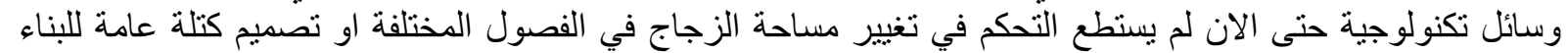

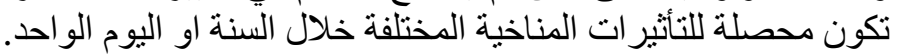

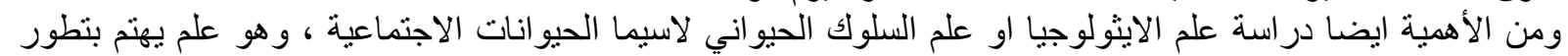

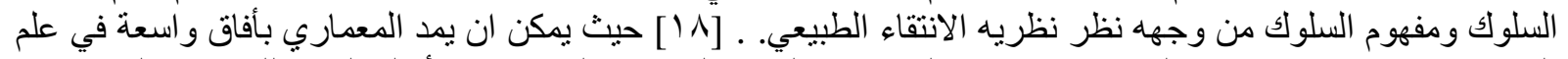

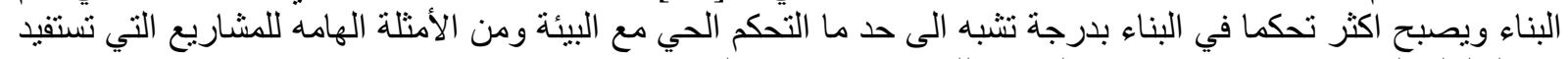

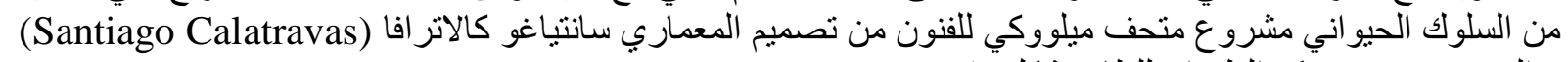
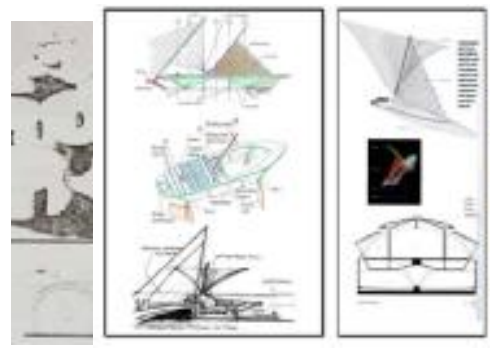

شكل (؛) هيكل متحف ميلووكي على شكل أجنحة ، الجنار

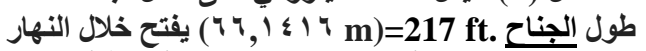
ويتقوس في الليل وأثناء العو اصف. هذه الأجنحة أصبحت

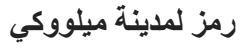

و المستوحى من حركه الطبر ان للطائر شكل (ع ع )
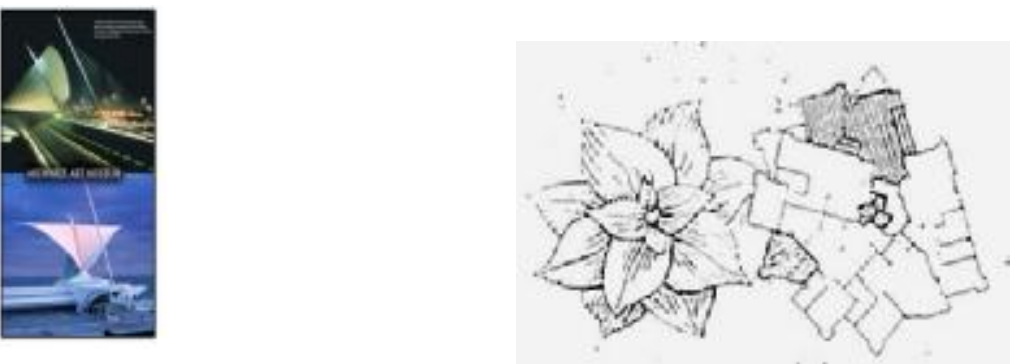

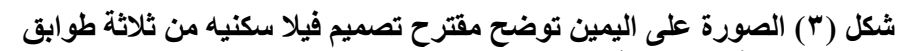

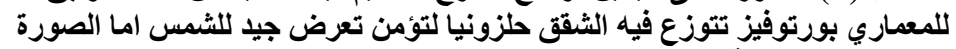

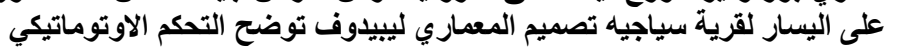

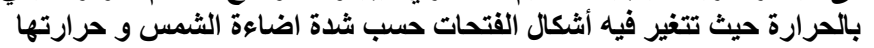




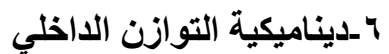

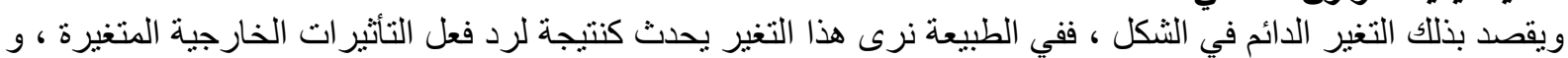

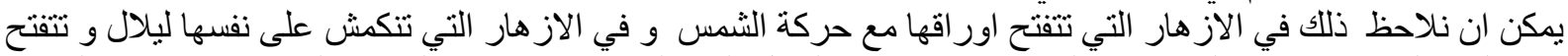

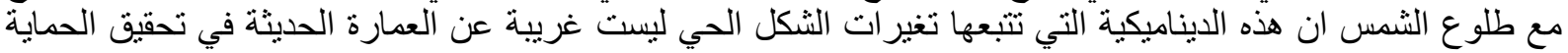

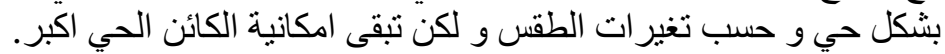

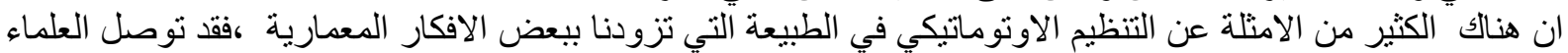

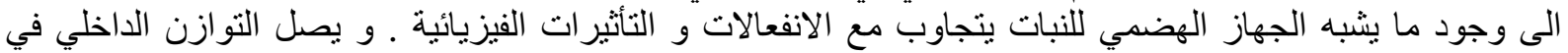

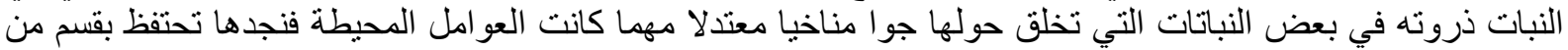

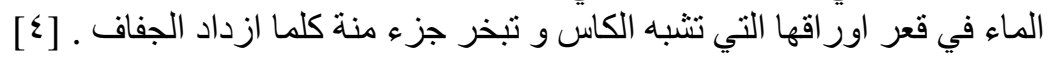

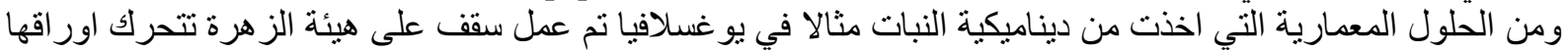

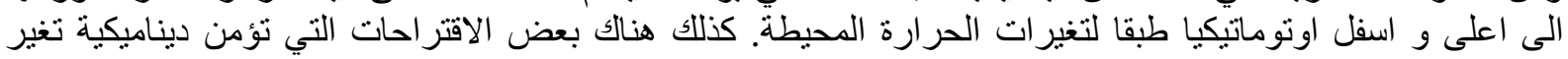

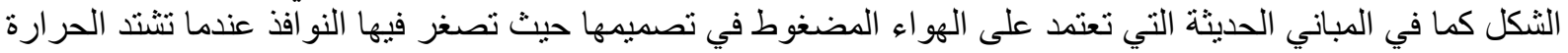
و و تكبر في الجو البارد .

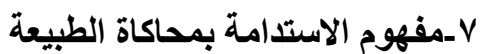

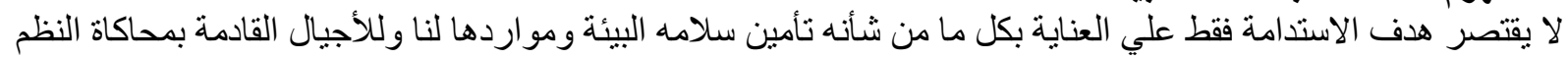

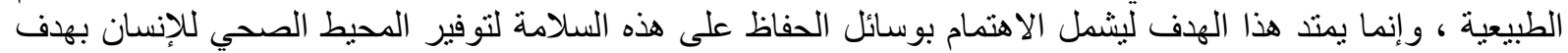

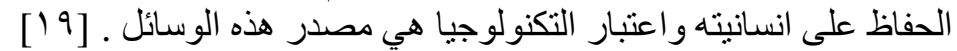

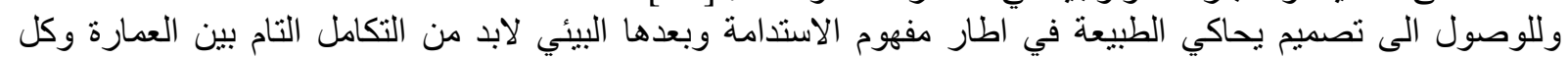

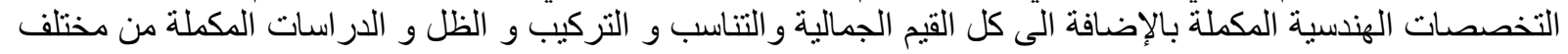

النواحي البيئية و الاقتصادية و البيئية وذلك باستخدام احدث التقنيات و التكنولوجيا الحديثة.

^ـمفهوم التصميم البيئي المستدام بمحاكاة الطبيعة

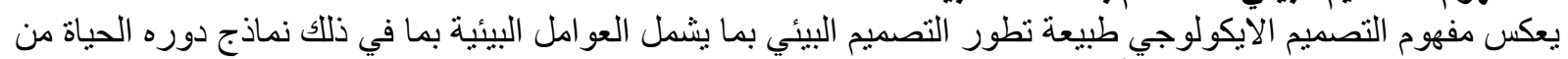

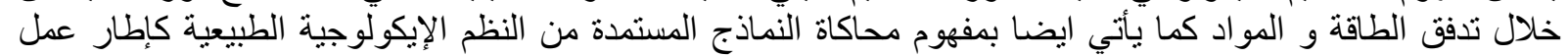

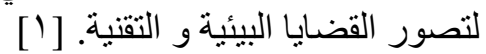

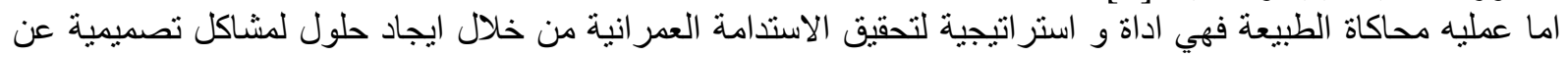
طريق المحاكاة ويتم ذللك من خلال عمليه تقليد للكائنات و الاشكال الموجودة بالطبيعة ومحاكاة نماذجها وآلية عملها

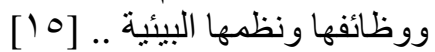

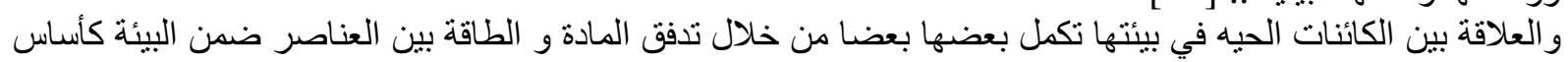

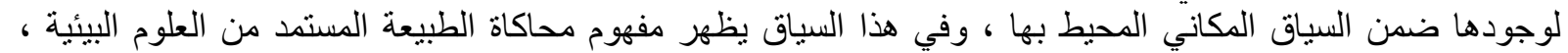

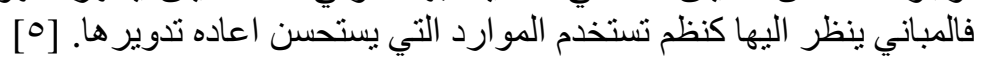

9 ـالمداخل الفكرية لمحاكاة الطبيعة

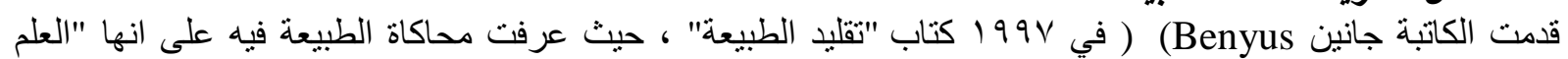

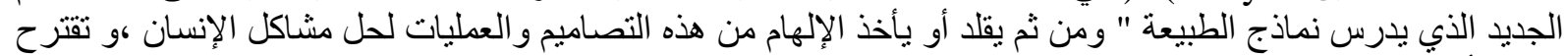

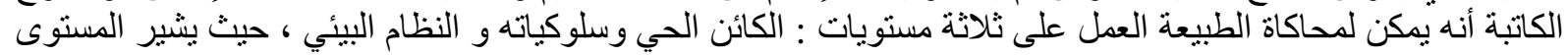

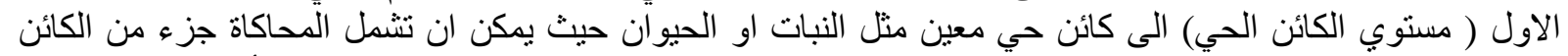

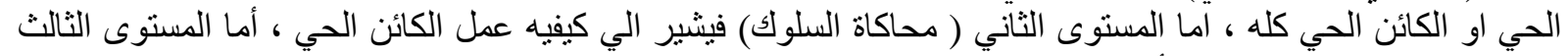

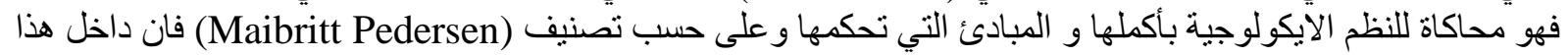

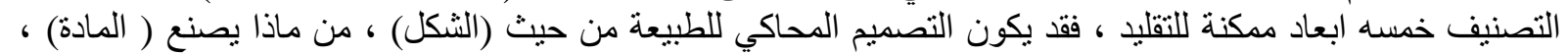

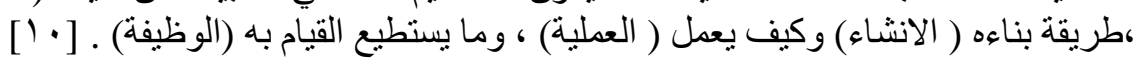

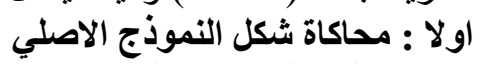

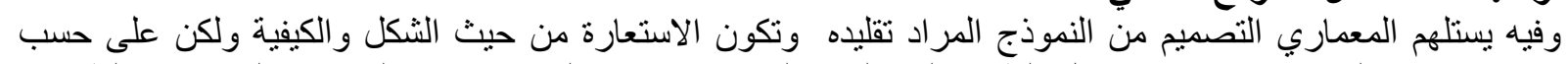

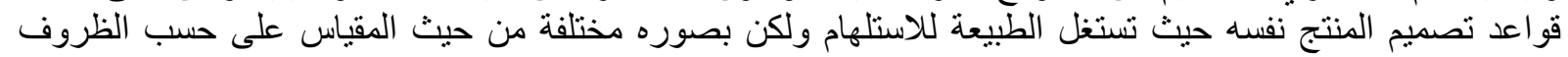

البيئية و المناخية. [11) مثال ا : برجولة مصممه ومستوحاة من شكل الاشجار والغرض من هذا التصميم هو التقاط صور الحيو انات مع نوفير

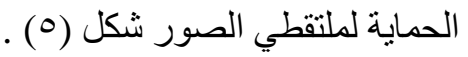




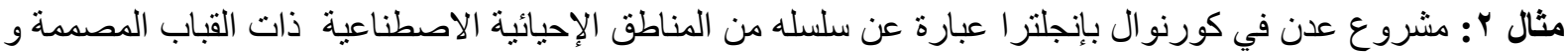

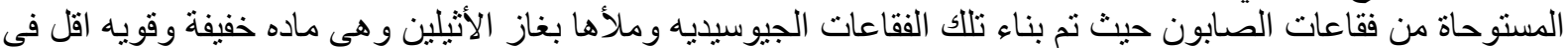

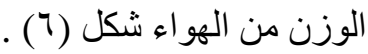

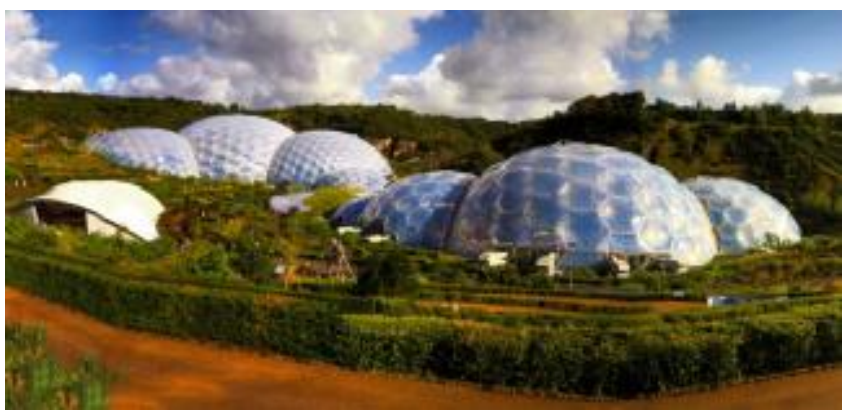

شكل ( 7 ): مشروع عدن في كورنوال مستوحى من فقاعات الصابون

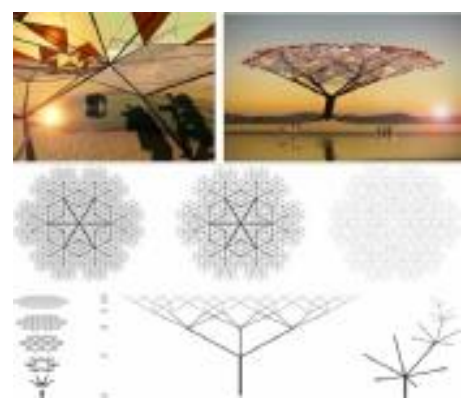

شكل ( •): برجولا مصممه ومستوحاة من شكل الاشجار .

\section{مثال بّ: مركز بكين الوطني للألعاب المائية}

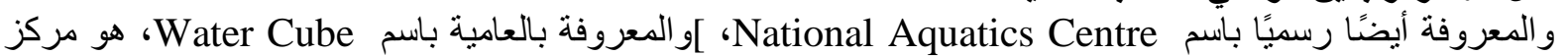

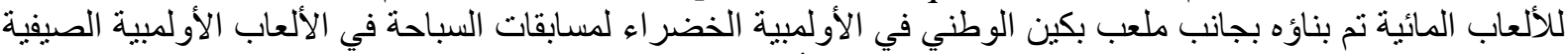

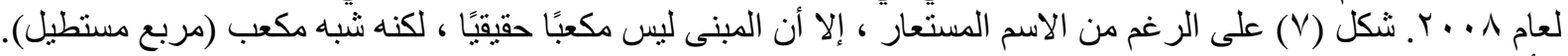

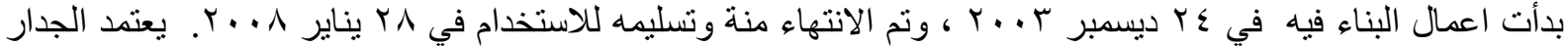

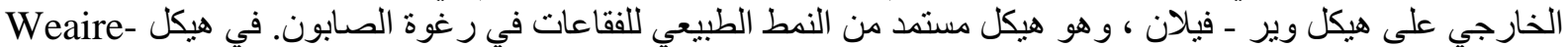
Phelan

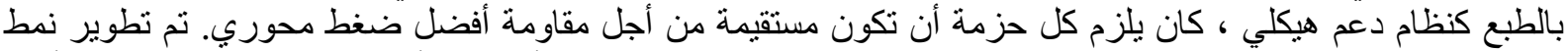
Weaire-Phelan

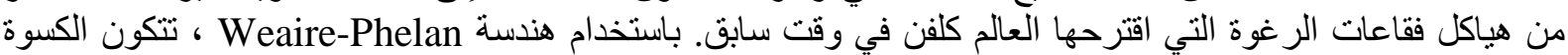

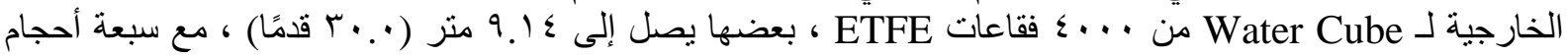

مختلفة للسقف و 10 اللجدران. [1']

\section{مثال ؛ : مركز تاييه المالي}

بالإنجليزية (Taipei) :كما يعرف أيضا باسم مركز تايييه المالي "Taipei Financial Center" ناطحة

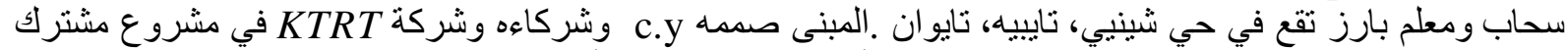

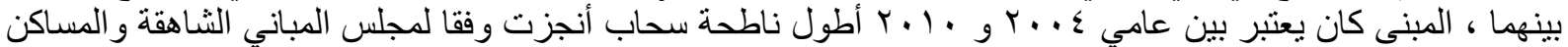
الحضرية.

يتضمن البناء 1 ـ الطابق فوق الأرض وه أسفلها ، بأسلوب يجمع بين الأوروبي الحديث و الآسيوي التقليدي ، وهي

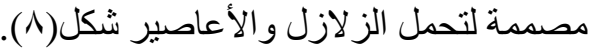

يتميز المبنى بالأفكار الغريبة المتبعة عند تصميمه و المستمدة من افكار بسيطة استخدمت في حز ام الامان في السيارات وكذلك التو ازن المستخدم بالقو ارب اضافة إلى الثنكل المستوحى من نبات قصب السكرة السكر.

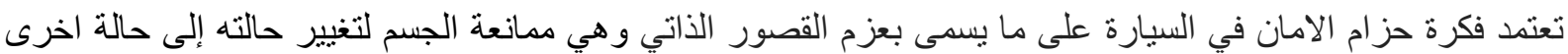

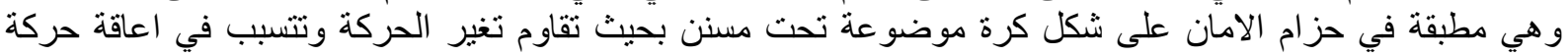

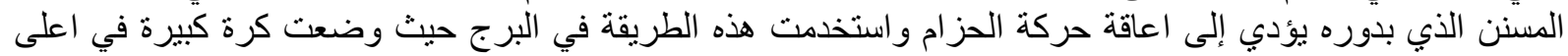

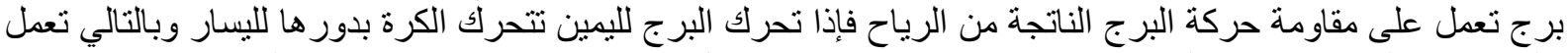

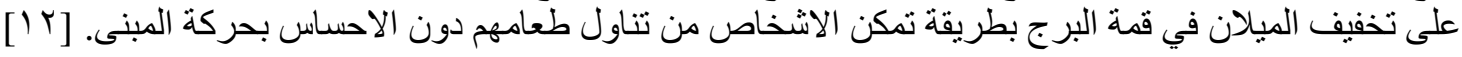

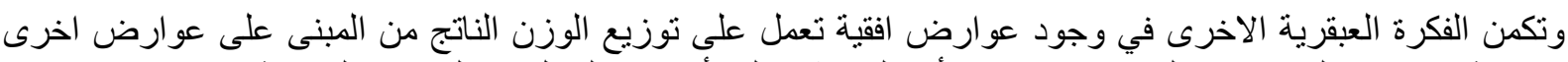

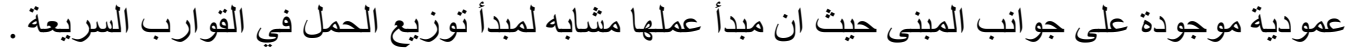


كما ان شكل المبنى مستمد من شكل نبات قصب السكر حيث ان المبنى يقاوم الانحناء بشكل كبير وذلك لوجوده على شكل

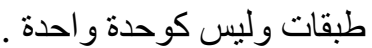

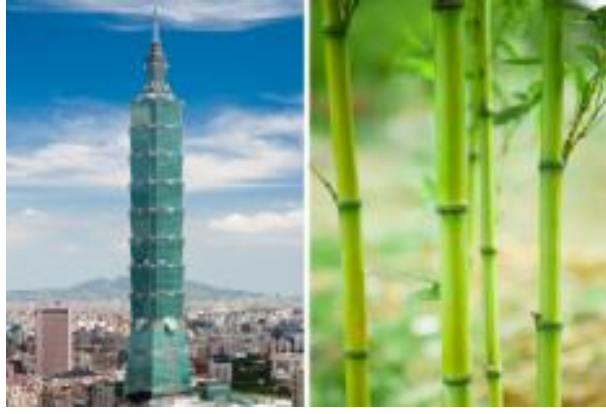

شكل (^): مركز تاييه المالي مستمد شكله من شكل نبات قصب
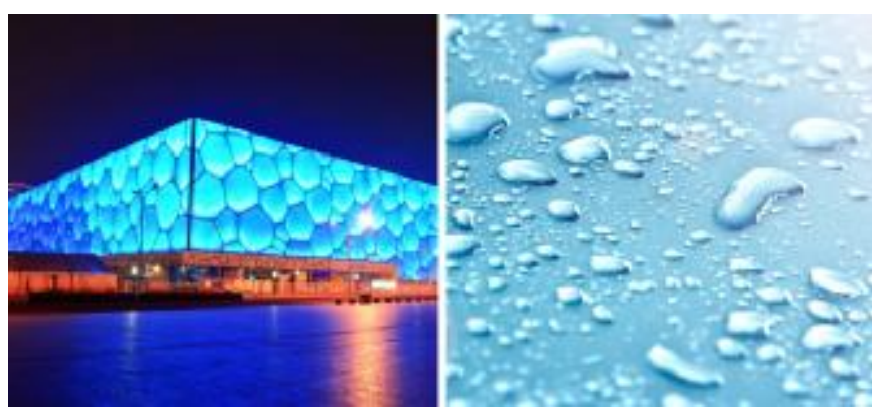

شكل (V): مركز بكين الوطني للألعاب المائية وتغطيه بهيكل يشبه فقاعات الصابون

مثال ه : تمثال السمكة لفرانك جيري

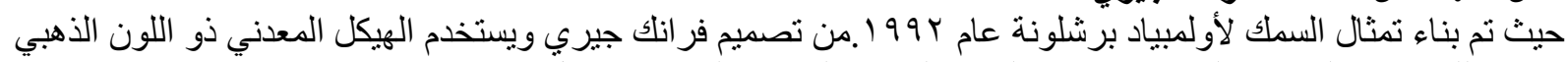

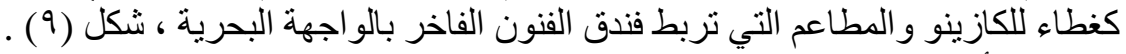

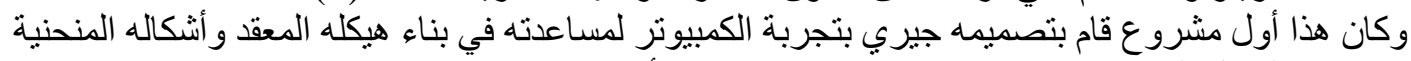
ويقع هذا الهيكل التذكاري في ميناء Olympic ، بجوار الأبر اج و Mapfre Arts Barcelona

المبني عبارة عن شبكة من الخطوط المعدنية الرفيعة تتقاطع لتشكيل هيكل سمكه بأسلوب تجريدي بشكل ملحوظ. ومع ذلك

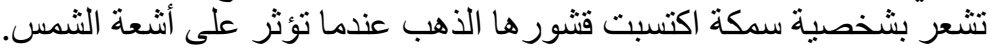

ويصف جيري الفكرة التصميمية لمنشآته "السمكة". بأنة هناك هيكل وأعضاء داخليه للأسماك تمنحها شكلها الديناميكي

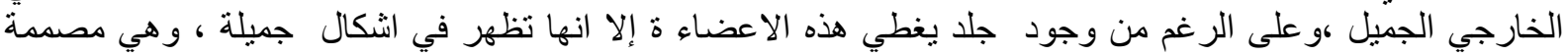

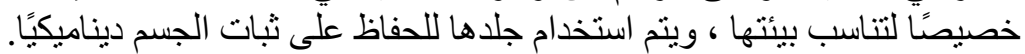

كما يستعين Gehry في التصميم بالكمبيونر لتوفير مو اصفات دقيقة لأشكالها الغير منماثلة خاصة عند البناء

مثثال 7 : قبه شنغهاي القلكية Shanghai Planetarium

بدأ المعماري Ennead Architects في بناء القبة السماوية في شنغهاي ، وهو مشروع جديد تبلغ مساحته . . . مب متر

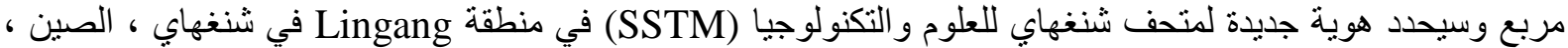

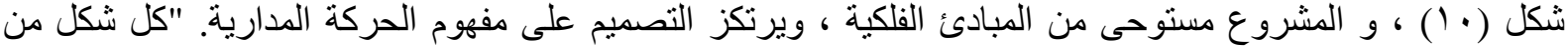

الأشكال الثلاثة الرئيسية للمبنى ـ العين والقبة المقلوبة والكرة - يعمل كأداة فلكية ويتتبع الثمس و القمر و النجوم. [Y l l]
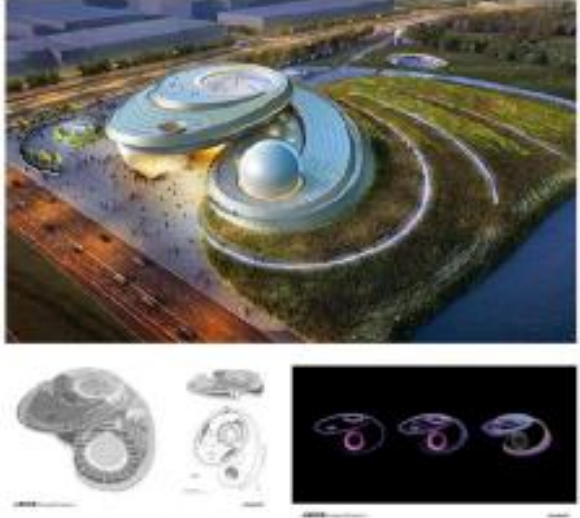

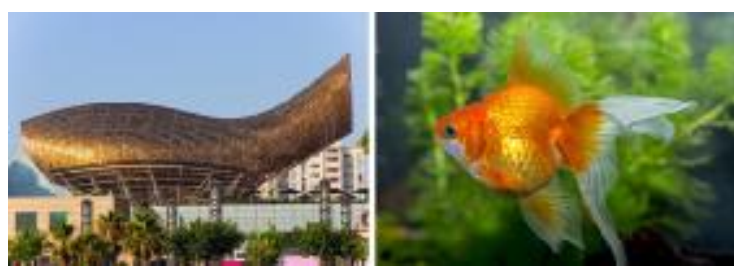

شكل ( 9): مشروع تمثال السمكة لفراتك جيري ذو بناء هيكلي وأشكاله منحنية

شكل ( • (1): مشروع قبه شنغهاي الفلكية مستوحى من المبادئ الفلكية 
ثانيا محاكاة سلوك الكائن الحي ويتم محاكاة رد فعل الكائن الحي وكيف يتأي ولفاعل مع بيئته و الذي يمكن ان يتغير فيها سلوك هذا الكائن حتي يتكيف مع البيئة ضمن حدود الطاقة وتو افر المو اد و هناك امتله عديدة عن هذا السلوك من المحاكاة .

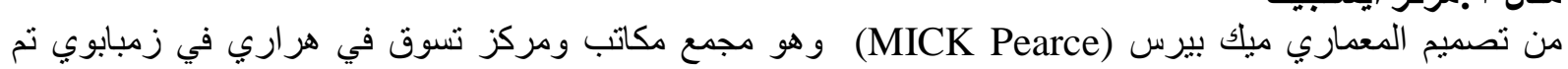

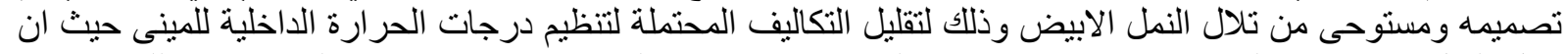

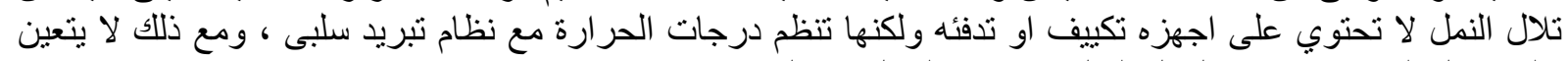

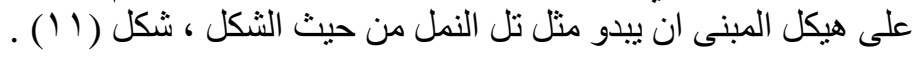

مثال ؟ : مبنى الصبار الفطري

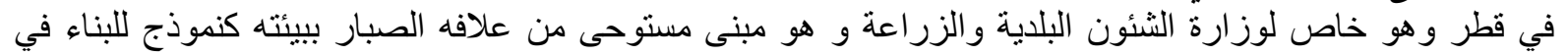

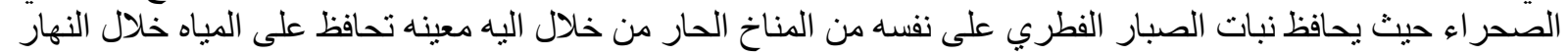

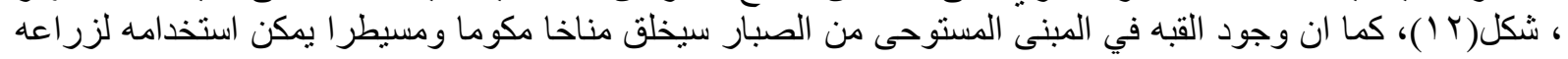

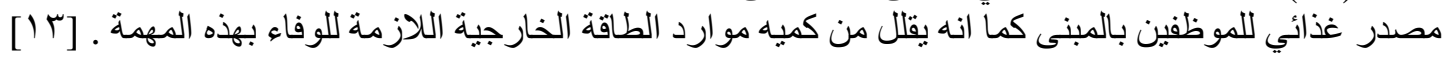

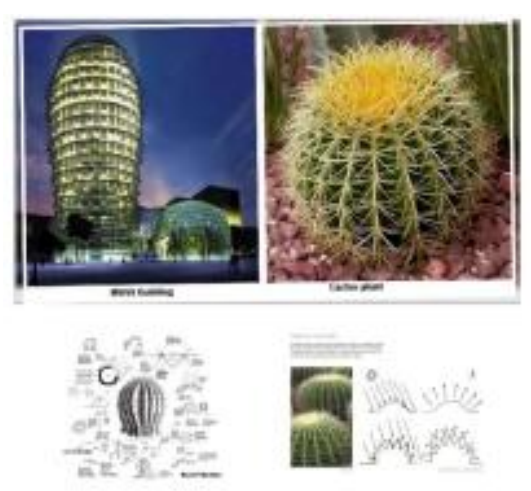

شكل (r 1 ): وزارة الثئون البلاية والزراعة مستوحى من نبات الصبار

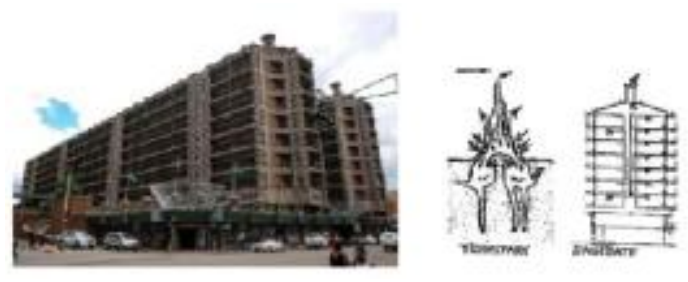

شكل (1 (1): مركز ايستجيت مجمع مكاتب مستوحى من تل النمل

ثالثا : محاكاة النظام البيئي

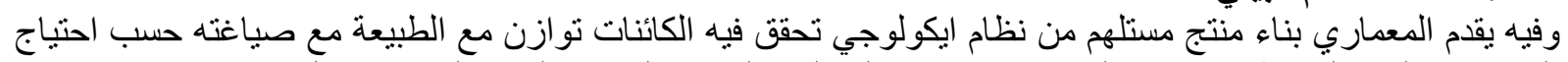

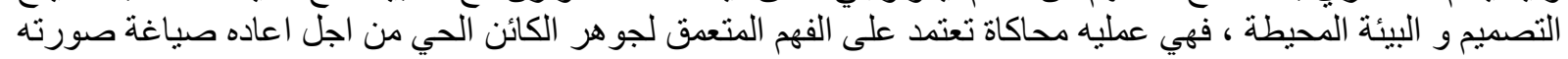

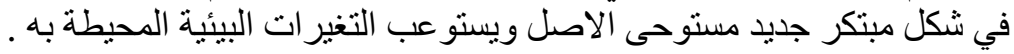

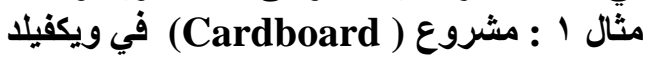

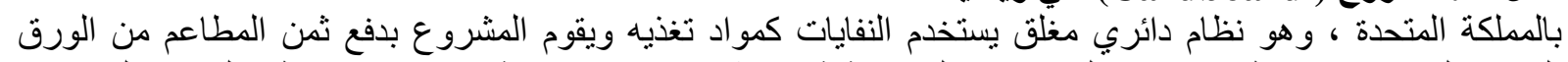

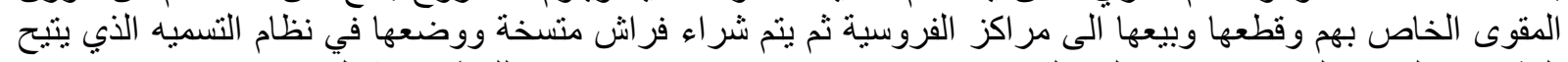

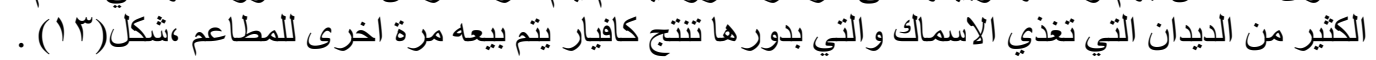

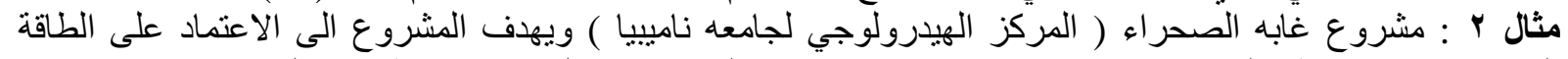

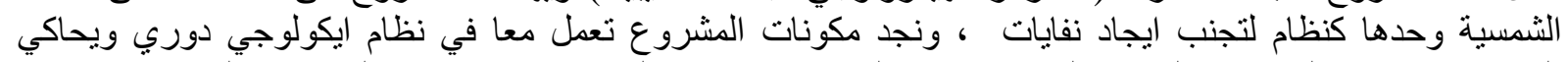

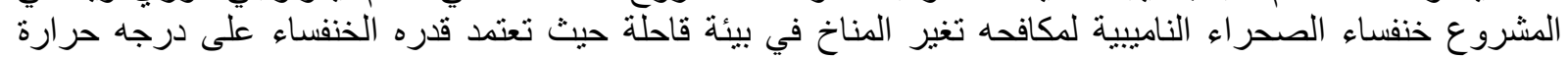

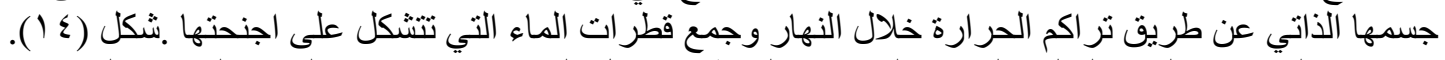

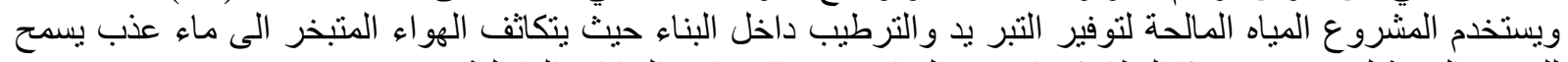

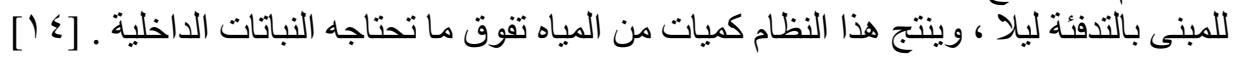



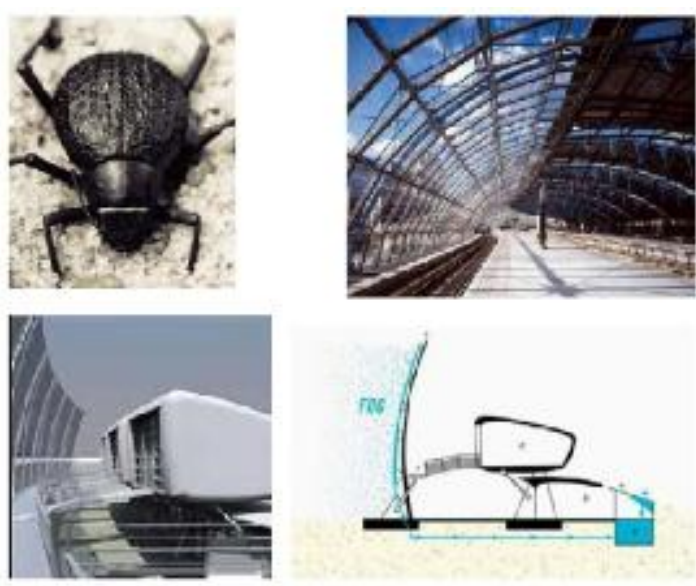

شكل ( \& 1 ) : مشروع غابه الصحراء نظام بيئي يحاكي خنفساء الصحر غاءه

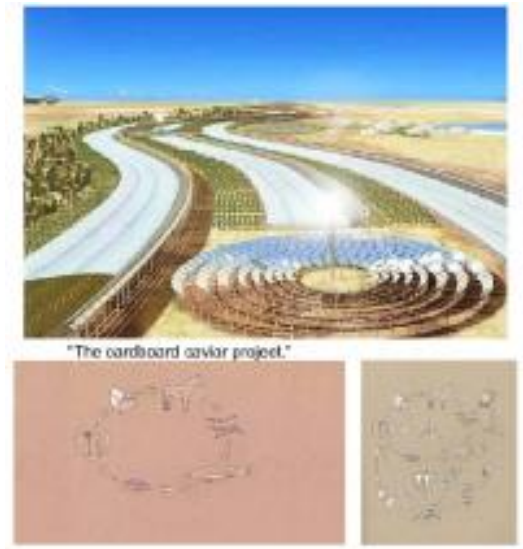

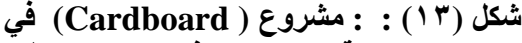
ويكفيلد بالمملكة المتحدة : نموذج لمحاكاة النظام البيئي

مثال ץ : برج نورمان فوستر برج (Swiss Re Tower)

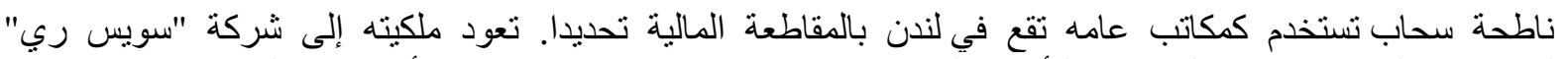

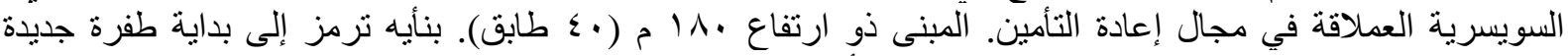

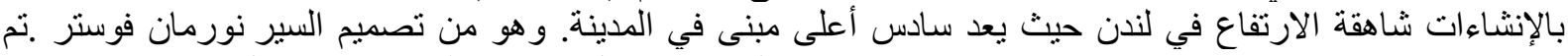

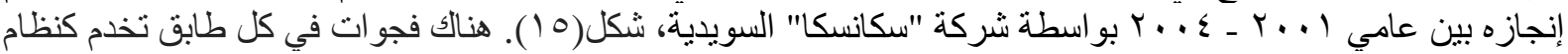

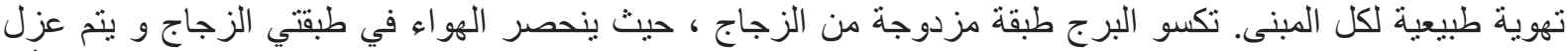

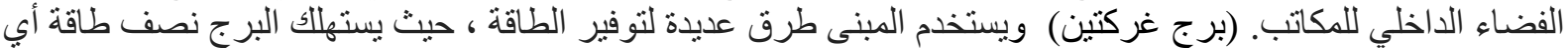

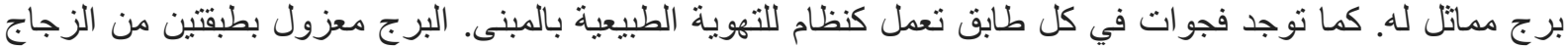

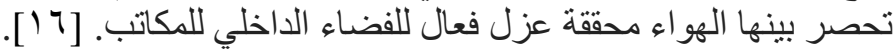

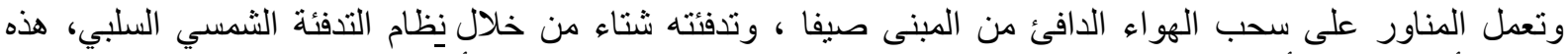

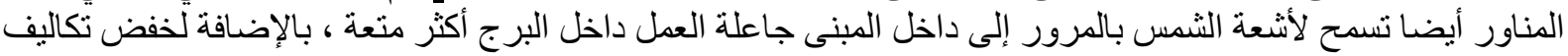

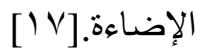

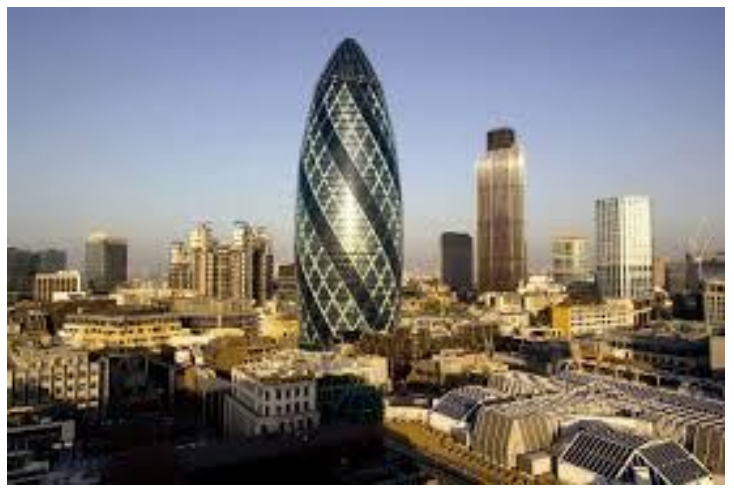

شكل (0 1 ) : برج المعماري نورمان فوستر (برج غركتين)

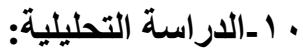

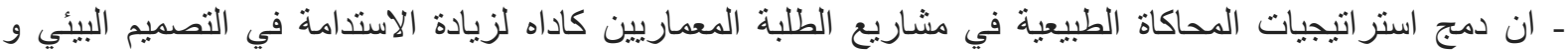

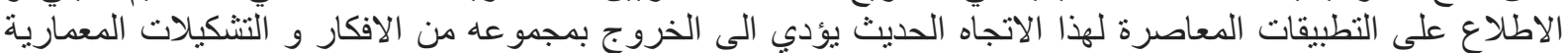

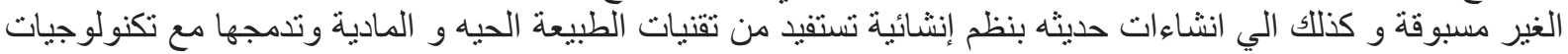

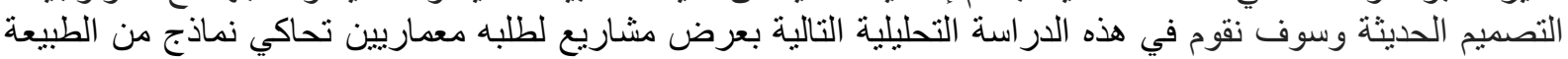
وتستخدم استر اتيجيات مستوحاة من هذه النماذج و سلوكها مع بيئتها 


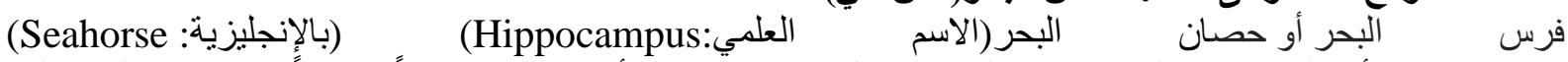

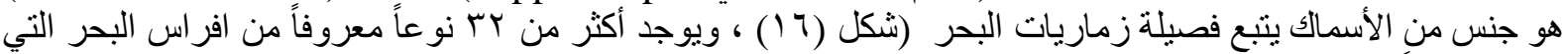

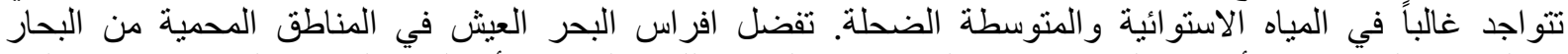

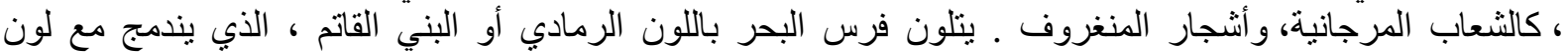

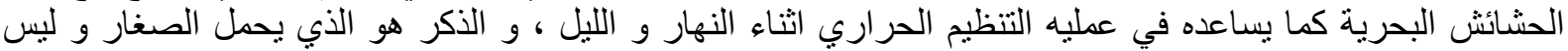

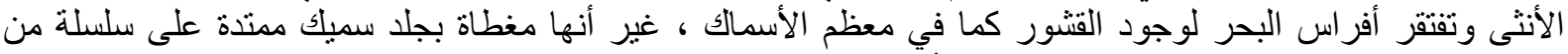

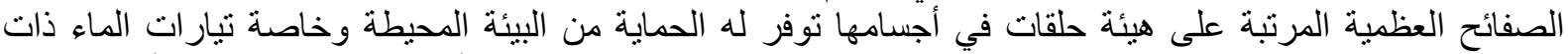

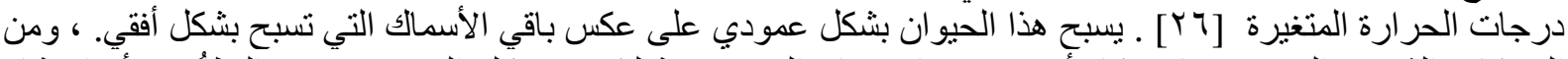

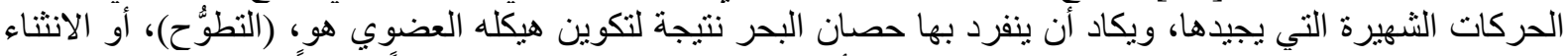

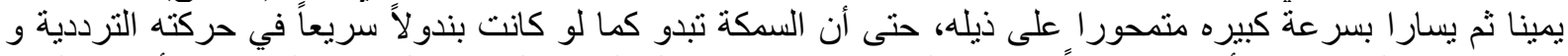

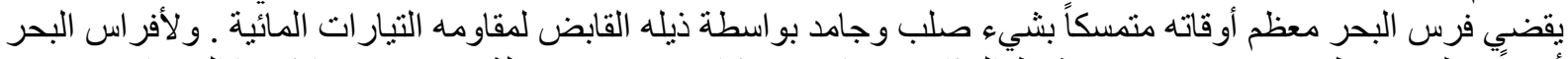

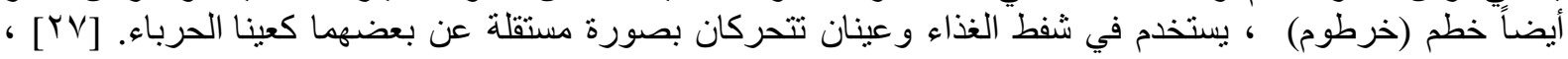
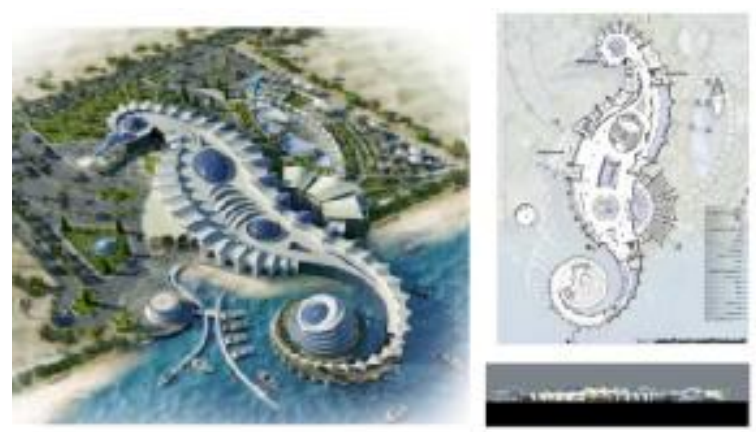

شكل (IV) : مشروع متحف مائي مستوحى من شكل حصان البحر

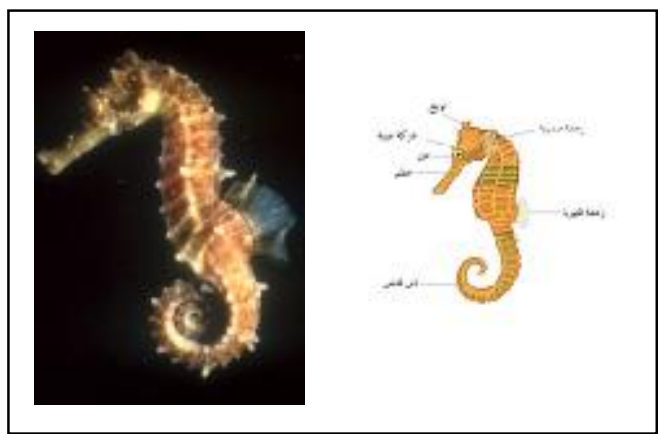

شكل ( 14) - 19) : حصان البحر كائن بحري

-1 .

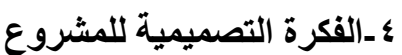

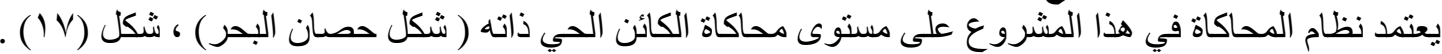

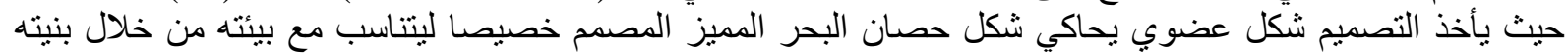

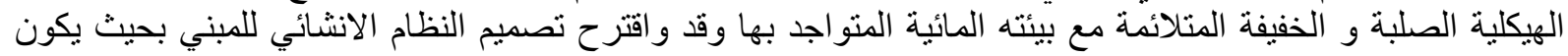

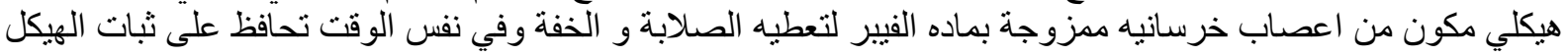

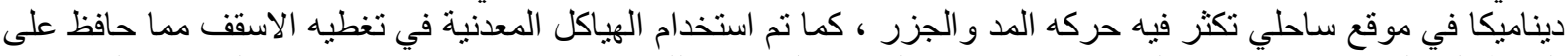

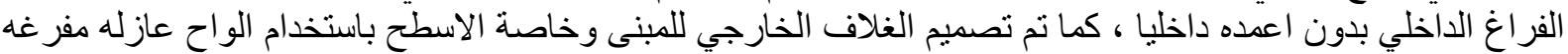

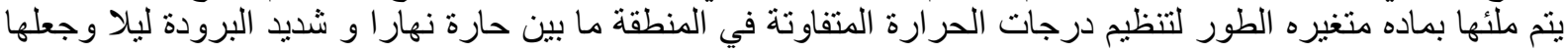
مستقرة طو ال اليوم . مانغيره

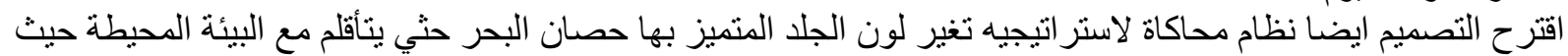

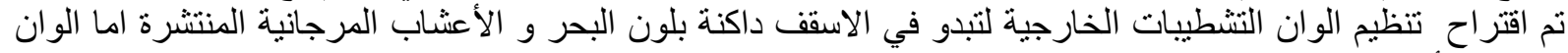

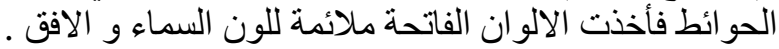

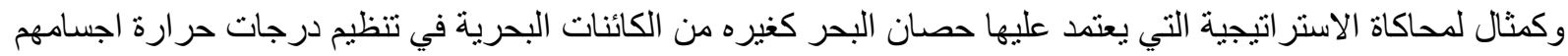

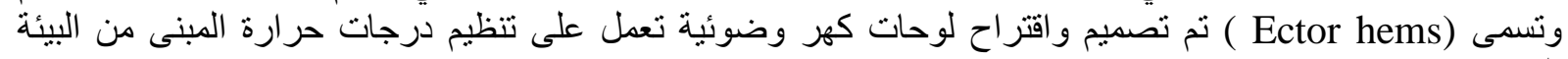

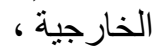

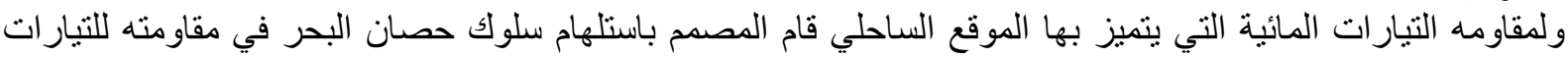

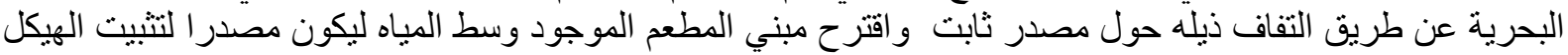
الخارجي ذو الشكل الرمزي لحصان البحر. 
11 إلمشروع الثاني : مركز ابحاث مائية

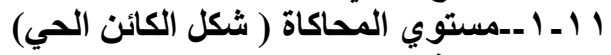

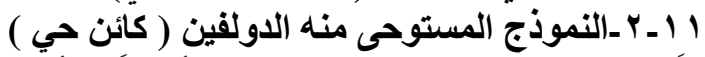

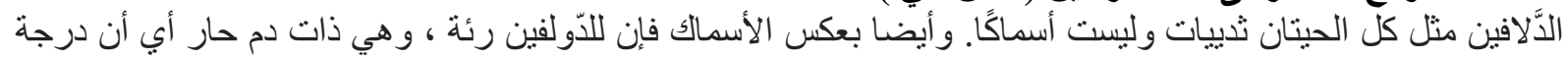

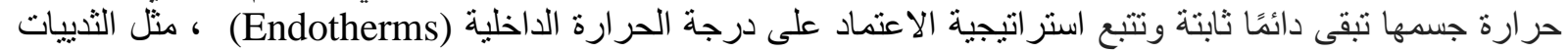

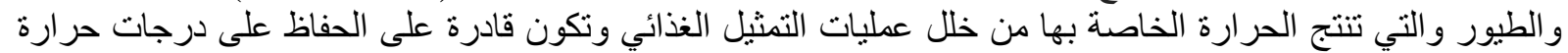

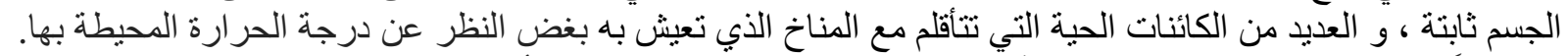

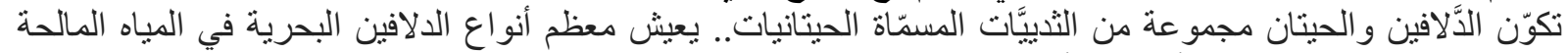

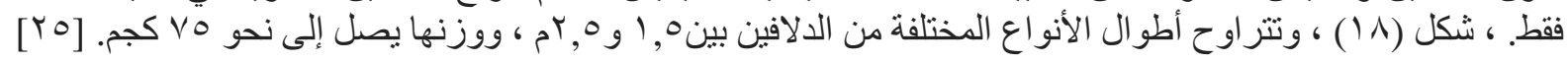

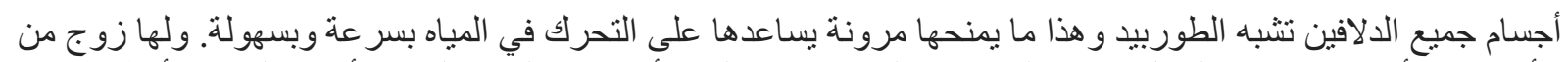

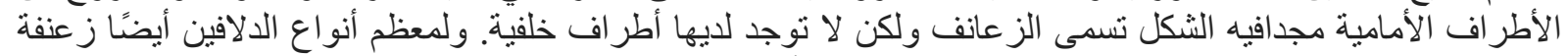
ظهرية على ظهر ها تساعد في المحافظة على اتزان الحئ الحيو ان أثناء العوم.
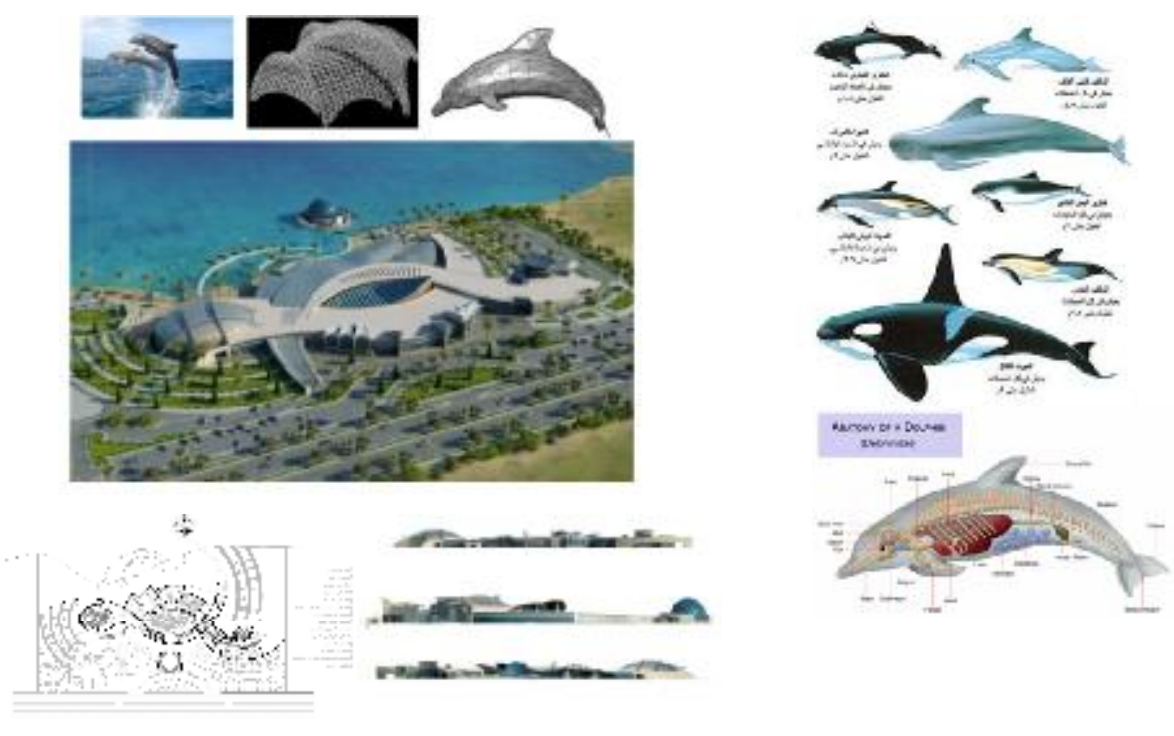

\section{شكل (9 1 ) : مشروع مركز ابحاث مائية كنموذج للمحاكاة على مستوى شكل الكائن الحي كي}

شكل (11) : الاولفين كائن بحري نموذج للمحاكاة بمستوى شكل الكائن الحي

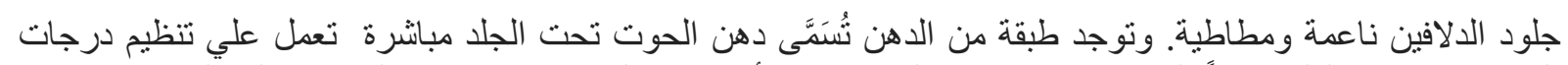

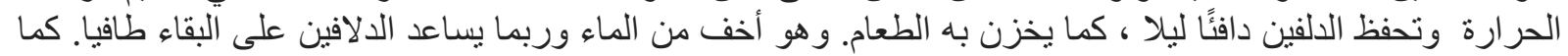

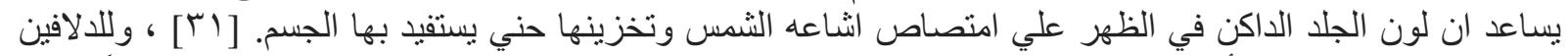

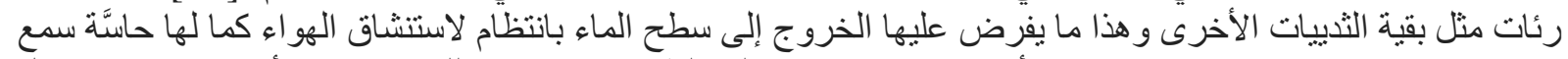

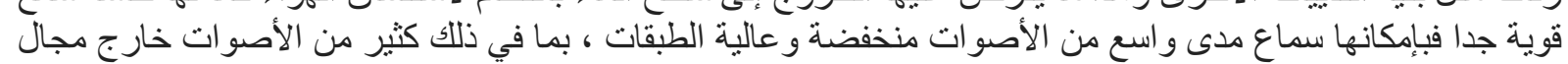

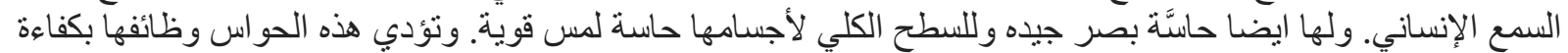

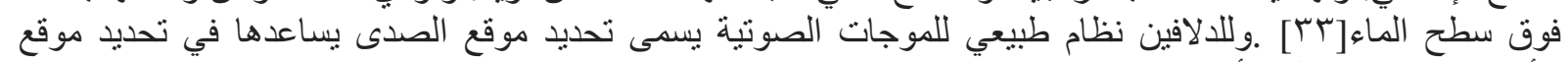
الأشياء تحت سطح الماء أثناء عومهاء

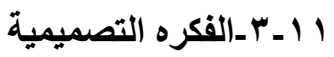

يعتمد نظام المحاكاة في هذا المشروع على مستوى شكل الكائن الحي حيث بعتبر البنيه العضوية المرنة و الهيكل العظمي

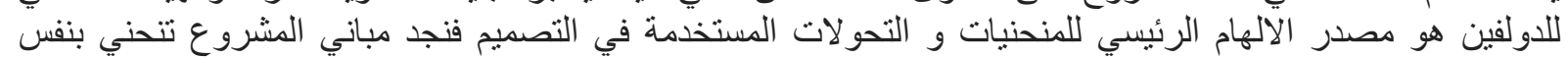

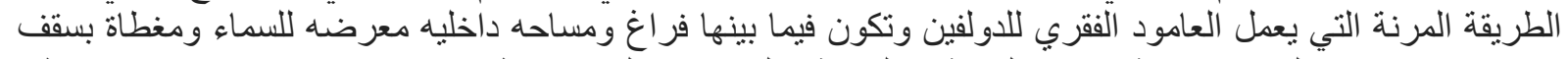

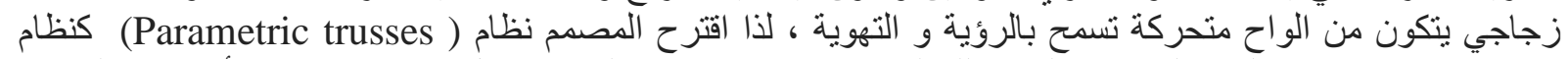

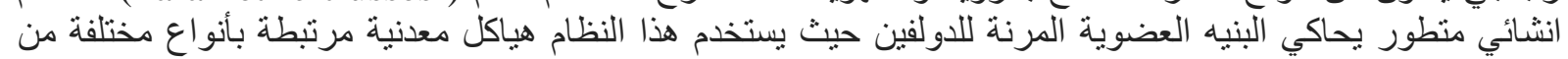

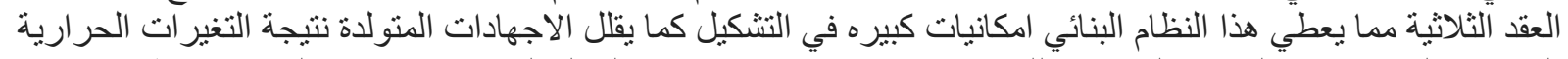

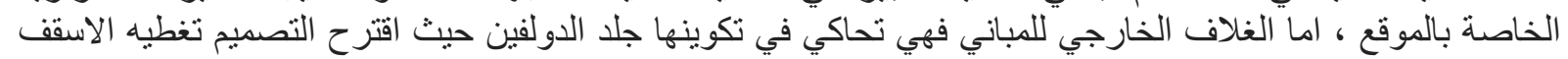




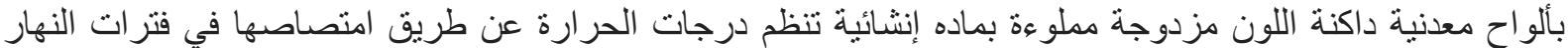

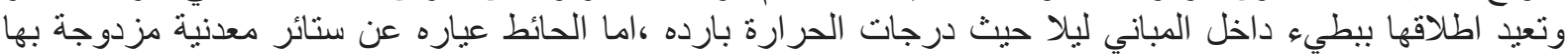

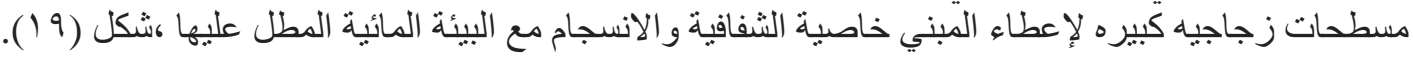

r r المشروع الثالث : مشروع قبة فلكيه (Planetarium ) r ا ـ ا ـ المستوي المحاكاة ( النظام البيئي )

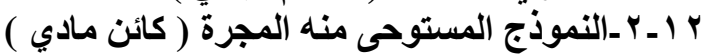

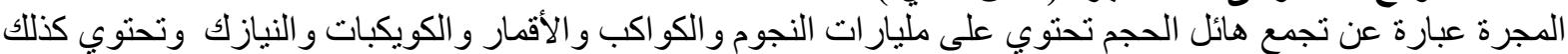

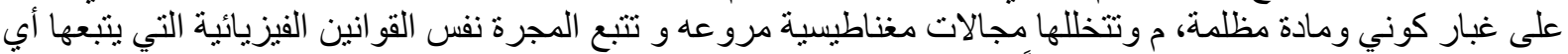

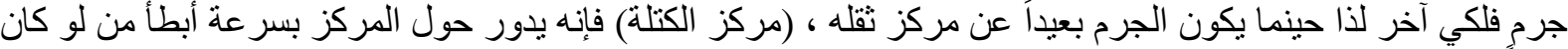

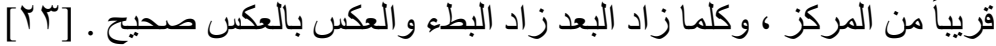

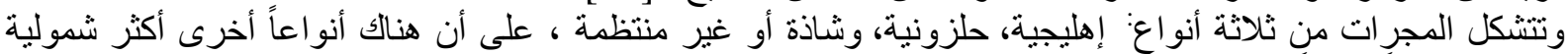

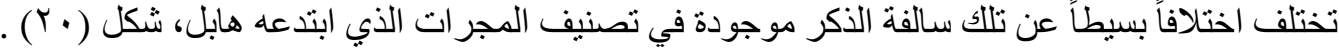

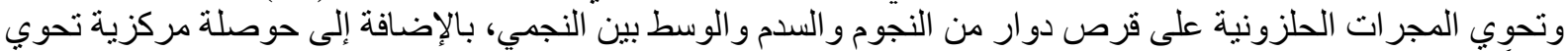

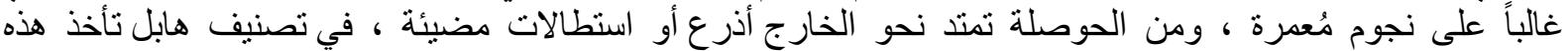

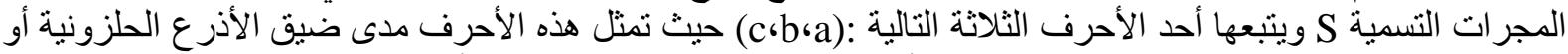

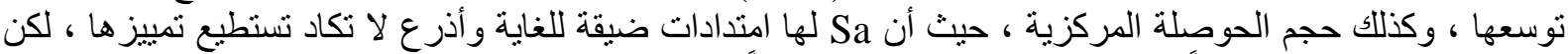

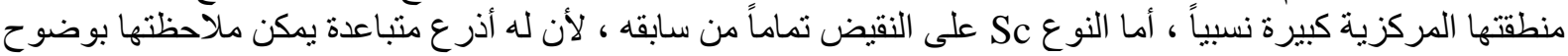

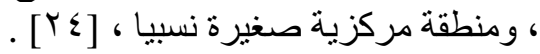
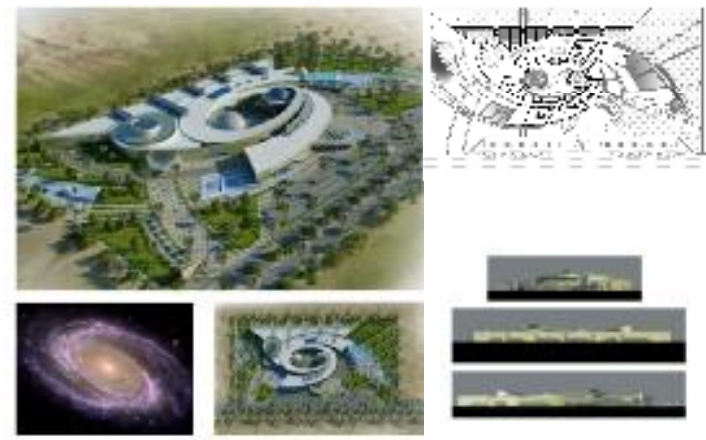

شكل ( M ا (Y) ) : مشروع قبه فلكيه كنموذج للمحاكاة

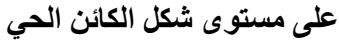

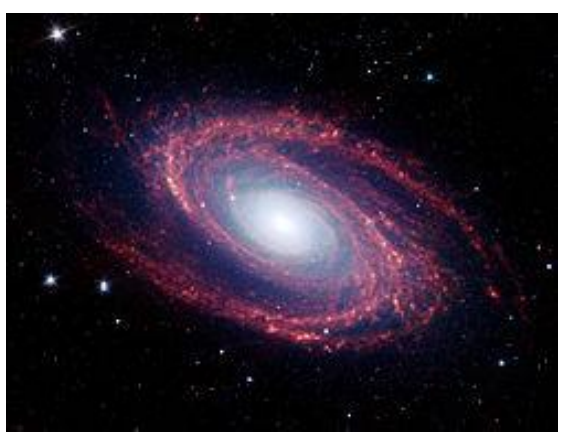

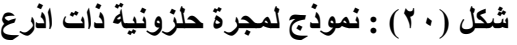

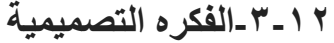

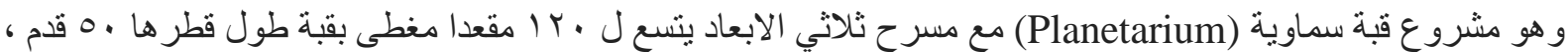

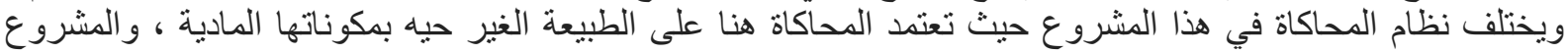

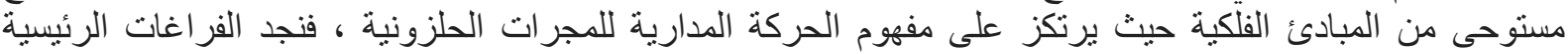

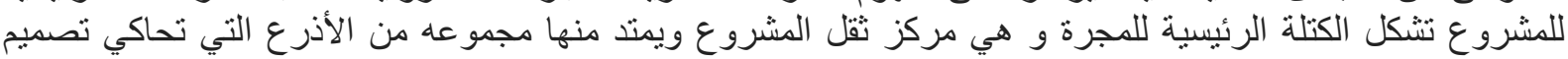

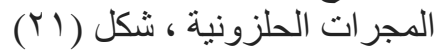

وتدور فكره المحاكاة عن تحقيق الاتزان في صوره مركزيه بين فر اغات المشروع الرئيسية و علاقته بين باقي الفراغات

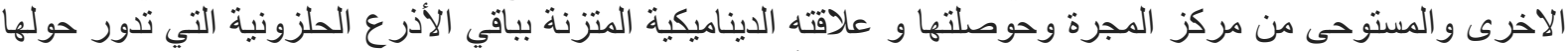

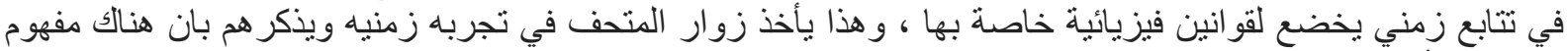

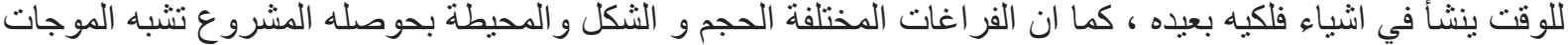
التي تحدث في ملاعب كره القدم عندما يرفع المشجعين ايديهم و يخفضو هاو وققا لتسلسل معين على شكل موجها.

كما اقترح التصميم ايضـا بعض الافكار الإنشائية التي تستخدم تقنيات الطبيعة وتتو افق مع مبادئ التصميم الفلكي من حيث

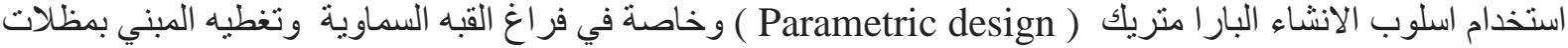

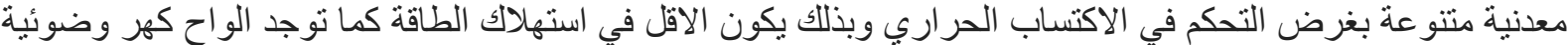

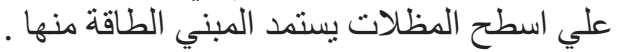


بالإضافة الي الفكرة الإنشائية للمبني قام المصدم باقتر اح العديد من التقنيات الفضائية التي تعتمد علي ظاهرة المحاكاة

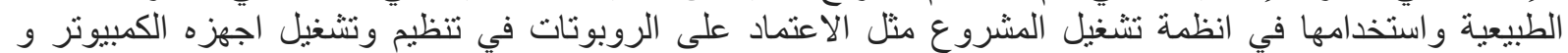

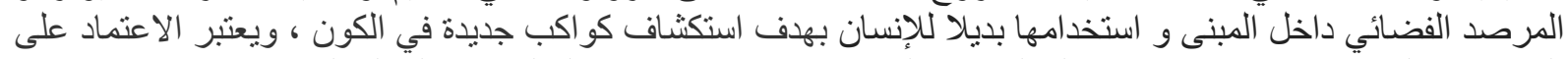

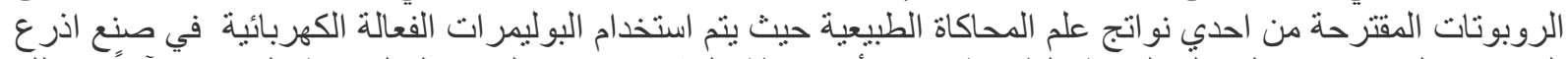

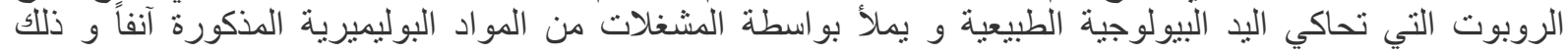

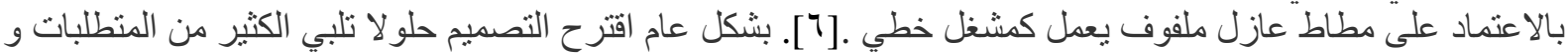

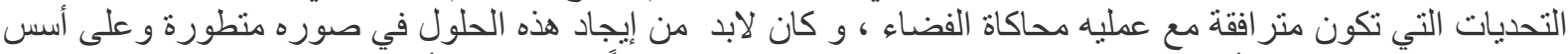

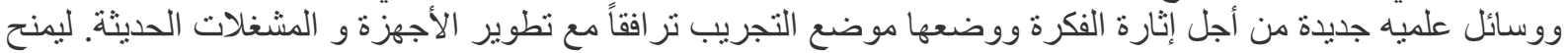

الزوار تجربه مثيره من اكتشاف الكو اكب وربما القيام برحلات فضائية مستقبليه .

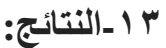
يوضح الجدول التالي نتائج تقييم وتحليل نماذج المشاريع التي اعتمدت على دمج استر اتيجيات المحاكاة الطبيعية في فكرتها التصميمية كاداه لزيادة الاستدامة في التصميم البيئي ، جدول (1) (1) .

جلول ( ) (1) يوضح نتائج تقييم مشاريع المحاكاة

\begin{tabular}{|c|c|c|c|}
\hline مشروع المحاكاة & كائن المحاكاة & مستوي المحاكاة & نتيجة المحاكاة \\
\hline & & شكل الكائن الحي & 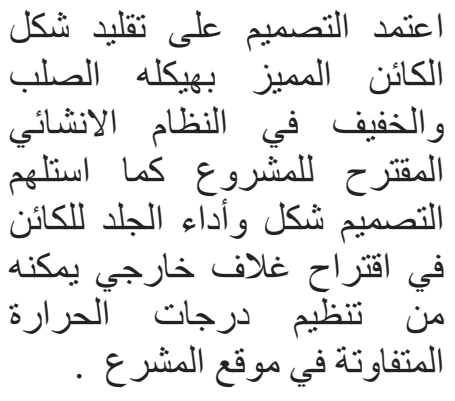 \\
\hline & & شكل وسلوك الكائن & 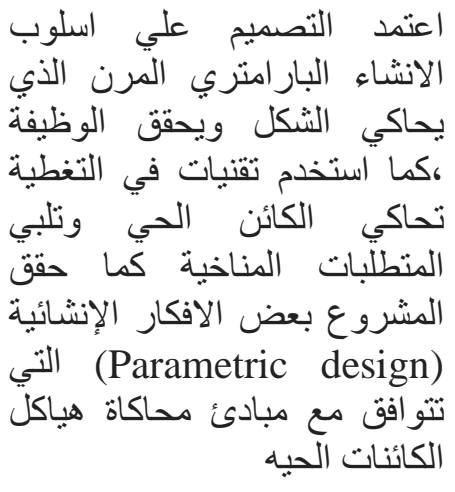 \\
\hline & & البيئي & 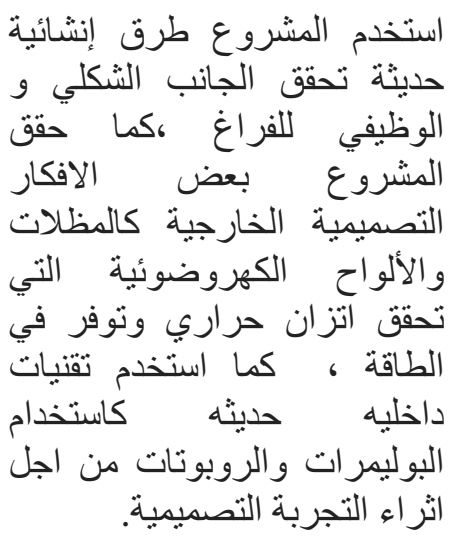 \\
\hline
\end{tabular}


- يعتبر علم المحاكاة الطبيعية و خاصة المحاكاة البيولوجية مصدر هام للفكر التصميمي و هذا العلم ينتج نتائج و انماط

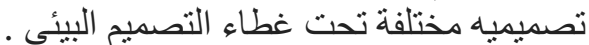
ـان الافكار التصميمية للعمارة المستدامة تتاح مباشره للتعامل مع النظم البيائية وغالبا ما يكون شكل المنتج النهائي مرتبط

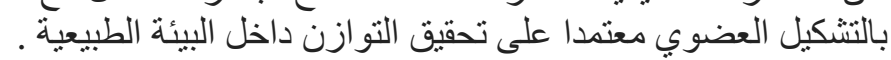

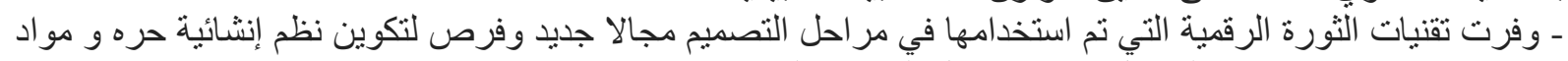

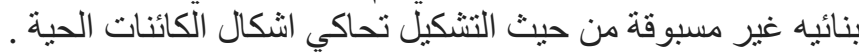

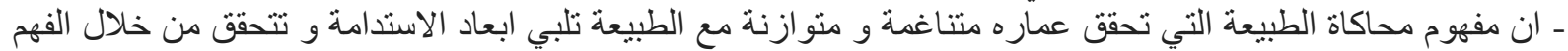

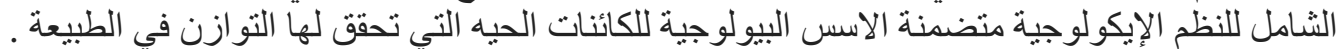
- يعتبر محاكاة النظام الايكولوجي للكائن الحي هو السياق الاشمل و الأوسع و و الذي يضم جميع مبادئ ونظم السياقات

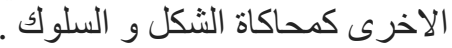
ـ تعتبر الهياكل المادية المقلدة كجزء من الطبيعة الغير حيه شبكه اكبر من البيئة الحيه او العضوية واكية وان هذه الهياكل و

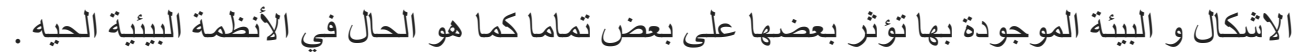

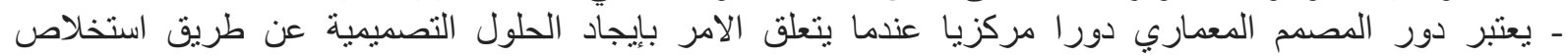

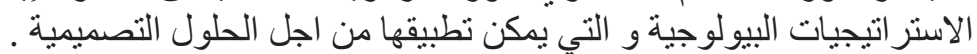

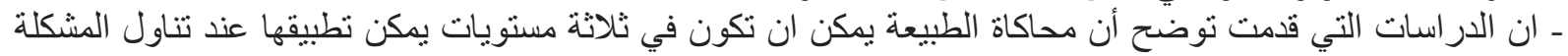

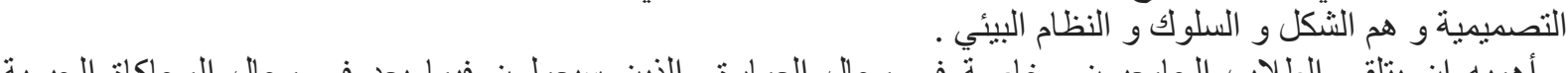

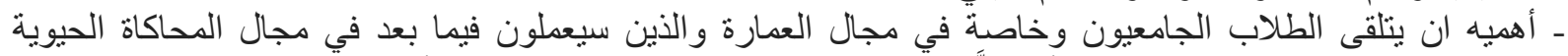

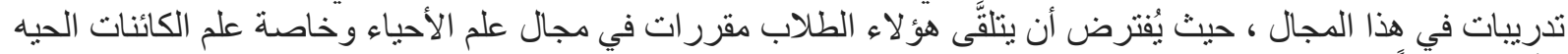

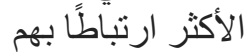
ـ الانتمام بالمحاكاة الحيوية أي محاكاة الصفات أو الأنظمة الحيوية في تطبيقات تمند من التصميمات المعمارية و المو اد إلى

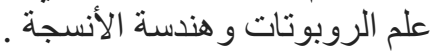

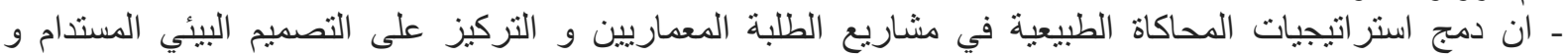

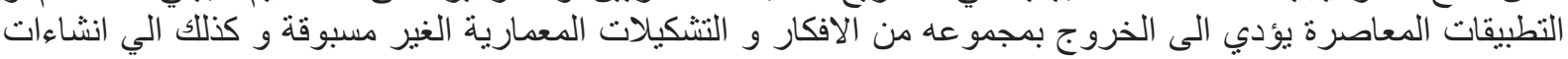

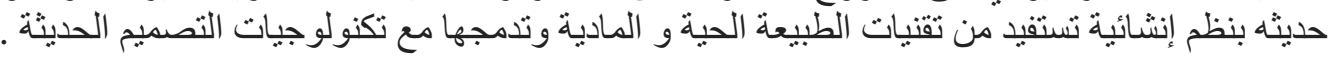

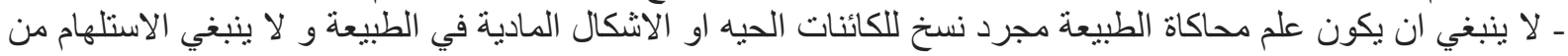

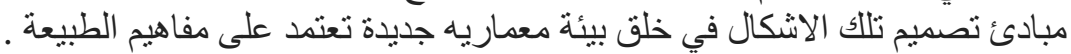

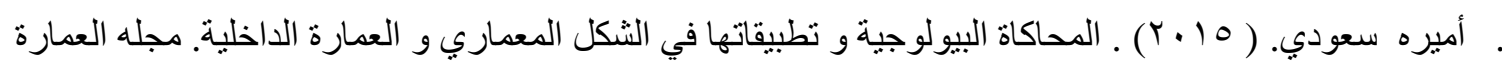

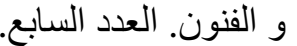

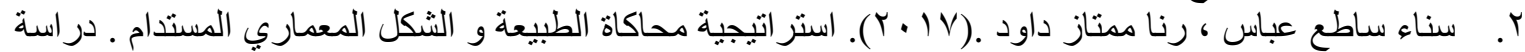

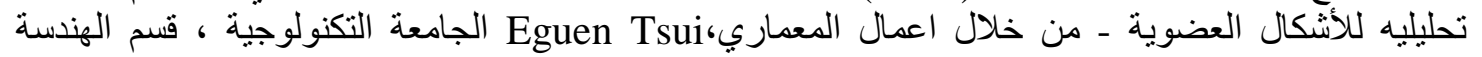
المعمارية.

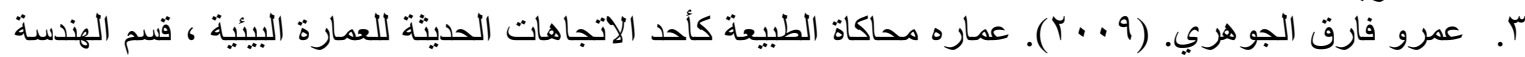

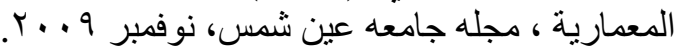

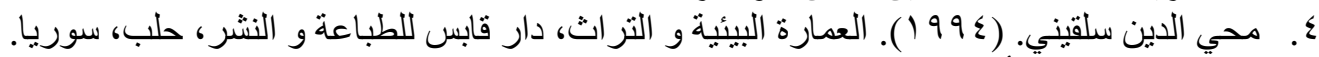

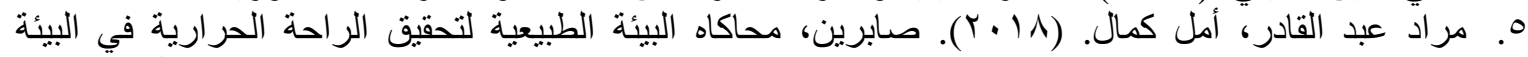

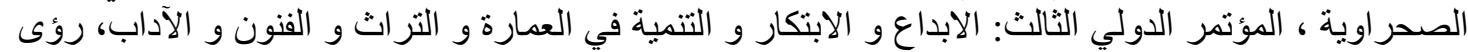

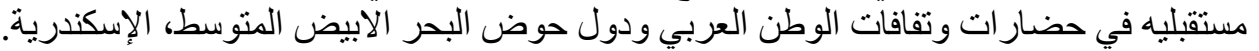

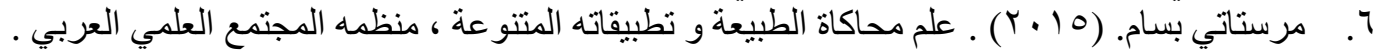

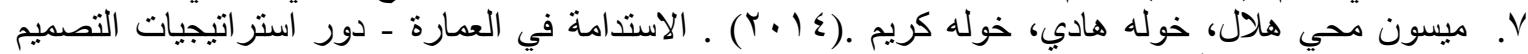

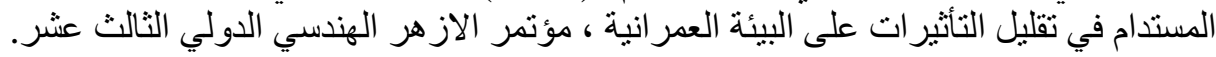

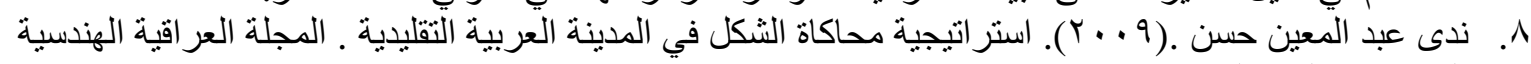

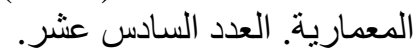

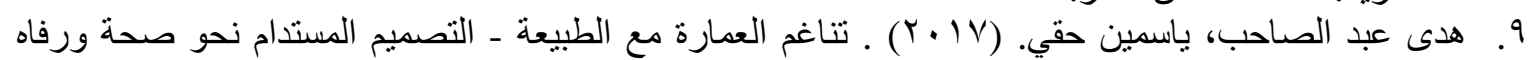

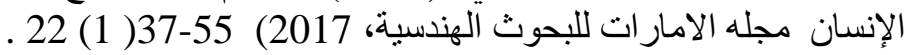


10. Ahmed.N. (2016), Biomimetic Principles role towards adaptive Building Envelope in Egypet, Master Thesis, Faculty of Fine Arts, Helwan University.

11. Benyus, J. (1997) Bio mimicry - Innovation Inspired by Nature, New York, Harper Collins Publishers.

12. Benyus, Janine. (1997). Bio mimicry: Innovation Inspired by Nature. New York, NY, USA: William Morrow \& Company, Inc.. ISBN 978-0688160999

13. Bio mimicry Guild (2007) Innovation Inspired by Nature Work Book, Bio mimicry Guild, April.

14. Charest, S. (2007). Ecosystem Principle Research, personal communication, May.

15. Elmeligy, D. A. (2016). Bio mimicry for ecologically sustainable design in architecture: a proposed methodological study. Eco-Architecture VI: Harmonisation between Architecture and Nature.

16. Kelth Evan Green.(2005). The "Bio-logic" Architecture (Environmental Design Inspired by Slime Mold, Lichen and Other Natural Sources), THE ART OF ARCHITECTURE/THE SCIENCE OF ARCHITECTURE • ACSA National 2005.

17. Lan Ma. (2014). Form Bionic Design of the High-Rise Buildings, Institute of Art \& Fashion Tianjin Polytechnic University, Tianjin, China

18. Mazzoleni, I. (2013). Architecture Follows Nature-Biomimetic Principles for Innovative Design (Vol. 2). Crc Press

19. Pedersen, Maibritt, Zari. (2007). BIOMIMETIC APPROACHES TO ARCHITECTURAL DESIGN FOR INCREASED SUSTAINABILITY, School of Architecture, Victoria University, PO Box 600, Wellington, New Zealand.

20. Rer.nat.Oliver, Phil,Gerd de Bruyn. (2015).Organism concepts in biology and architecture as the basis for an interdisciplinary synopsis of constructional biomimetic

21. Sheta, A. (2010). Bio mimicry in environmental architecture: Exploring the Concept and Methods Of The Bio-Inspired Environmental Architectural Design, Faculty of Engineering, Cairo University, MS.C.

22. -http://biomimicry.(http://biomimicry.net

23. - https://ar.m.wikipedia.org/wiki/ثجرون

24. - https://ar.m.wikipedia.org/wiki/ذراع حلزوني///2.

25. Https://Ar.Wikipedia.Org/Wiki/\%D8\%AF\%D9\%84\%D9\%81\%D9\%8A\%D9\%86

26. $\mathrm{Https}$ ://Ar.Wikipedia.Org/Wiki/\%D8\%AF\%D9\%84\%D9\%81\%D9\%8A\%D9\%86

27. $\mathrm{Https}$ ://Ar.Wikipedia.Org/Wiki/\%D9\%81\%D8\%B1\%D8\%B3 \%D8\%A7\%D9\%84\% $\mathrm{D} 8 \% \mathrm{~A} 8 \% \mathrm{D} 8 \% \mathrm{AD} \% \mathrm{D} 8 \% \mathrm{~B} 1$

28. Https://Mawdoo3.Com/ فو ائدحصان_البحر

29. فو ائدحصان_البحر/Zttps://Hyatok.Com

30. www.britannica.com, Retrieved 30-3-2018. Edited.

31. Jennifer Kennedy. (17-6-2017), "Why Whales are Mammals and Not Fish" ، www.thoughtco.com, Retrieved 30-3-2018. Edited .

32. James G. Mead.(30-3-2018). "Cetacean" 'www.britannica.com, Retrieved 30-3-2018.

33. David A. Duffus. (30-3-2018). "Whales" 'www.encyclopedia.com, Retrieved 30-32018. 